ARTICLE

\title{
Structural and biophysical correlation of anti-NANP antibodies with in vivo protection against P. falciparum
}

\author{
Tossapol Pholcharee (10 1, David Oyen 1,6, Yevel Flores-Garcia'², Gonzalo Gonzalez-Paez (D) 1, Zhen Han ${ }^{1,7}$, \\ Katherine L. Williams ${ }^{3}$, Wayne Volkmuth ${ }^{3}$, Daniel Emerling ${ }^{3}$, Emily Locke ${ }^{4}$, C. Richter King $\mathbb{1}^{4}$, \\ Fidel Zavala (i) ${ }^{2} \&$ lan A. Wilson (1) ${ }^{1,5}$ 凶
}

The most advanced $P$. falciparum circumsporozoite protein-based malaria vaccine, RTS, S/AS01 (RTS,S), confers partial protection but with antibody titers that wane relatively rapidly, highlighting the need to elicit more potent and durable antibody responses. Here, we elucidate crystal structures, binding affinities and kinetics, and in vivo protection of eight anti-NANP antibodies derived from an RTS,S phase $2 a$ trial and encoded by three different heavy-chain germline genes. The structures reinforce the importance of homotypic Fab-Fab interactions in protective antibodies and the overwhelmingly dominant preference for a germline-encoded aromatic residue for recognition of the NANP motif. In this study, antibody apparent affinity correlates best with protection in an in vivo mouse model, with the more potent antibodies also recognizing epitopes with repeating secondary structural motifs of type I $\beta$ - and Asn pseudo $3_{10}$ turns; such insights can be incorporated into design of more effective immunogens and antibodies for passive immunization.

\footnotetext{
${ }^{1}$ Department of Integrative Structural and Computational Biology, The Scripps Research Institute, La Jolla, CA, USA. ${ }^{2}$ Malaria Research Institute, Johns Hopkins Bloomberg School of Public Health, Baltimore, MD, USA. ${ }^{3}$ Atreca Inc, South San Francisco, CA, USA. ${ }^{4}$ PATH's Malaria Vaccine Initiative, Washington, DC, USA. ${ }^{5}$ The Skaggs Institute for Chemical Biology, The Scripps Research Institute, La Jolla, CA, USA. ${ }^{6}$ Present address: Pfizer Inc, San Diego, CA, USA. ${ }^{7}$ Present address: Wondfo USA Co., Ltd, San Diego, CA, USA. ${ }^{凶}$ email: wilson@scripps.edu
} 
M alaria is caused by unicellular eukaryotic Plasmodium parasites, and $P$. falciparum is responsible for most malaria morbidity and mortality. Despite significant progress over the past 20 years, resistance of mosquito vectors to pyrethroid $^{1}$ and the emergence of multidrug-resistant parasite strains ${ }^{2}$ emphasize the need for new tools, including vaccines, to combat the disease. The most advanced malaria vaccine candidate to date is the RTS,S/AS01 vaccine, which has completed phase 3 clinical trials in young African children and is currently undergoing a large scale pilot introduction in Malawi, Ghana, and Kenya to inform on a policy decision for broader use ${ }^{3}$. The vaccine is based on PfCSP, which densely covers the surface of sporozoites and plays a critical role in the $P$. falciparum life cycle from the development of sporozoites in the mosquito midgut to liver-stage development in humans $s^{4-7}$. The N-terminal domain of CSP includes a heparan sulfate binding site for hepatocyte adhesion ${ }^{4}$, followed by the immunodominant central repeat region $^{8}$, and the C-terminal $\alpha$-thrombospondin repeat ( $\alpha$ TSR) domain that contains multiple T-cell epitopes ${ }^{9}$. The repeat region in P. falciparum is composed of 1 NPDP, 3-5 NVDP, and 35-41 NANP repeats ${ }^{10-13}$. In contrast, RTS,S contains only 19 NANP repeats and the aTSR domain, linked to the hepatitis B surface antigen protein (HBsAg), and was expressed recombinantly with soluble HBsAg to form a virus-like particle that is administered with the AS01 adjuvant ${ }^{14}$. RTS,S displayed $~ 40 \%$ efficacy against clinical malaria disease over 4 years of follow-up in phase 3 clinical testing; vaccine efficacy is highest in the period immediately following immunization and declines coincident with decay of induced $\mathrm{Ab}$ titers to $\mathrm{CSP}^{15-18}$. A similar vaccine candidate, R21, composed only of the same HBsAg-CSP fusion (i.e., without extra HBsAg), and formulated with Matrix-M adjuvant, has recently entered phase 2 clinical testing and is showing comparable efficacy levels in early clinical studies ${ }^{19}$. Another candidate is the attenuated, whole-sporozoite-based PfSPZ vaccine ${ }^{20}$, delivered by direct venous inoculation. It primarily aims to induce cellular immunity, and is thus associated with lower antiCSP antibody titers compared to R21 and RTS,S; however, vaccine efficacy in endemic field studies has been modest ${ }^{21}$. These clinical studies highlight the need to improve current vaccines to induce either more durable protection and/or higher potency antibody responses.

Recently, many anti-CSP antibodies have been characterized using structural and biophysical approaches and various functional assays, which have contributed to our growing insights into humoral immune responses against CSP. The junctional region, which corresponds to the amino-acid sequence of PfCSP between the N-term domain and the NANP repeats, contains NPDP and NVDP motifs and has been shown as a target for potent antibody responses ${ }^{22,23}$. However, recent evidence suggests that antijunction mAbs can also cross-react with the NANP repeats, and their protective capacity can be correlated with their binding promiscuity to NANP 24,25 . Epitopes of anti-NANP antibodies typically contain 2-3 NPNA structural motifs, which can adopt local conformations of a type I $\beta$-turn and an Asn pseudo $3_{10}$ turn $^{22,23,26-30}$. Anti-NANP antibodies often utilize a germlineencoded Trp residue to interact with the Pro or Asn in the NPNA turns $^{29}$. Additionally, some of these Abs exhibit unusual homotypic inter-Fab contacts when they simultaneously recognize the adjacent repeating epitopes on the NANP region of $\mathrm{CSP}^{26,27}$. Despite these structural and biophysical advances, the implications for antibody and immune responses, especially which properties or structural features correlate with in vivo protection by anti-NANP antibodies, are still not resolved.

In this work, we characterize eight monoclonal antibodies $(\mathrm{mAb})$ derived from protected volunteers who participated in a phase 2a clinical trial of RTS,S/AS01 ${ }^{31}$ with a delayed fractional dosing regimen, and compare them with three previously published mAbs derived from the same clinical trial ${ }^{28,29}$. Our data suggest a correlation between antibody apparent affinity (driven by the off-rate) and in vivo protection, which could serve as an important basis for subsequent characterization and engineering of anti-NANP mAbs. Two antibodies exhibit homotypic Fab-Fab interactions, which increase avidity to the repeat peptides as found in previous studies, and may have implications for $\mathrm{Ab}$ responses against CSP as an unusual type of antigen ${ }^{26,27}$. Cocrystal structures also reveal conserved and convergent use of aromatic residues for interaction with the NANP repeat region. Furthermore, we observe that binding to NANP repeats with an extended conformation, which lacks any secondary structural motifs, may not be optimal for stable antibody interaction and could contribute to low affinity to CSP and, subsequently, poor protection. On the other hand, potent and high-affinity antibodies all recognize epitopes containing a type I $\beta$-turn and/or Asn pseudo $3_{10}$ turn. These two structural motifs are observed as the repeating unit on a soluble recombinant shorter version of CSP (rsCSP) that can then adopt an unusual, long-range, extended spiral conformation in the presence of such antibodies $^{27}$. Altogether, these comprehensive characterizations of anti-NANP antibodies enhance our understanding of human humoral immune responses against CSP and provide a strong foundation for the design of next-generation malaria vaccines.

\section{Results}

Many anti-NANP antibodies show potent functional activity in vivo. The sequences of the antibodies in this study were originally derived from samples from protected volunteers in a phase 2a trial of RTS,S/AS01 seven days after the third fractional dose, as previously reported ${ }^{27-29}$. Antibodies were selected for study here from among the expanded sequence families with a focus on prevalent $I G H V$ families. The eight monoclonal antibodies (mAbs) chosen for study were encoded by three different immunoglobulin heavy variable (IGHV) genes and recombinantly expressed. These mAbs were compared to previous mAbs 311,317 , and 397 from this set that included two other IGHV genes $^{27-29}$. Experiments to evaluate in vivo protection were conducted for two panels of antibodies using two mouse models that assess parasite liver burden load and blood-stage parasitemia $^{32,33}$. The first panel contained $\mathrm{mAbs}$ derived from the IGHV3-33 gene (mAb239, 337, 356, 364, and 395), and the second consisted of mAbs encoded by IGHV3-49 (mAbs 224, 399), IGHV1-2 (mAb366), IGHV3-15 (mAb397), and IGHV3-30 (mAb317) germline genes, with $\mathrm{mAb311}$ (from IGHV3-33) as a control across both experiments. To assess reduction of parasite liver burden load, mice $(N=5)$ received $100 \mu \mathrm{g} \mathrm{mAb}$ by intravenous injection (IV), which is a dose established previously for standard comparison of anti-NANP antibodies ${ }^{32,33}$, and after 16 $\mathrm{h}$, mice were challenged with chimeric sporozoites $(P$. berghei sporozoites expressing full-length $P$. falciparum CSP) (Fig. 1a). All antibodies significantly reduced parasite burden (except for mAb395 $(p<0.05)$; $p<0.01$ for other mAbs, Mann-Whitney U test) with most mAbs inhibiting parasite development by at least 93\% (Fig. 1a). However, in the first panel, mAb395 performed significantly worse and mAb337 showed less in vivo protection compared to the others $(p<0.01$, Mann-Whitney U test) (Supplementary Table 1). In the second panel, mAbs 366 and 397 also displayed weaker protection, which was significantly less than mAbs 224 and $399(p<0.05$, Mann-Whitney U test) (Supplementary Table 1).

To further validate the liver burden results, $\mathrm{mAbs}(100 \mu \mathrm{g}$ or $300 \mu \mathrm{g})$ were passively transferred by IV to mice $(N=6) 16 \mathrm{~h}$ before exposure to bites of infected mosquitoes as in previous 
a Parasite liver-burden load $(100 \mu \mathrm{g})$

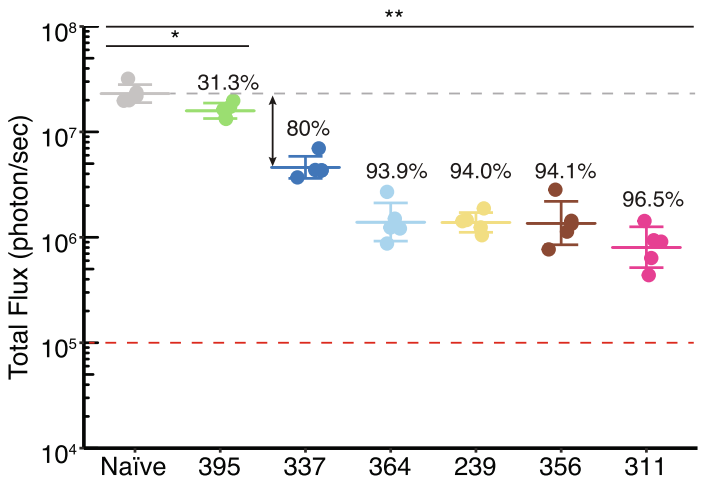

b Blood-stage parasitemia $(300 \mu \mathrm{g})$

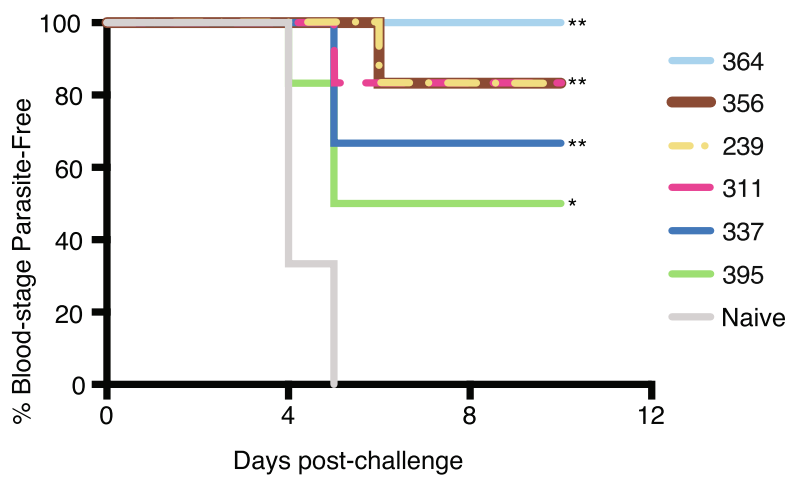

c Blood-stage parasitemia $(100 \mu \mathrm{g})$

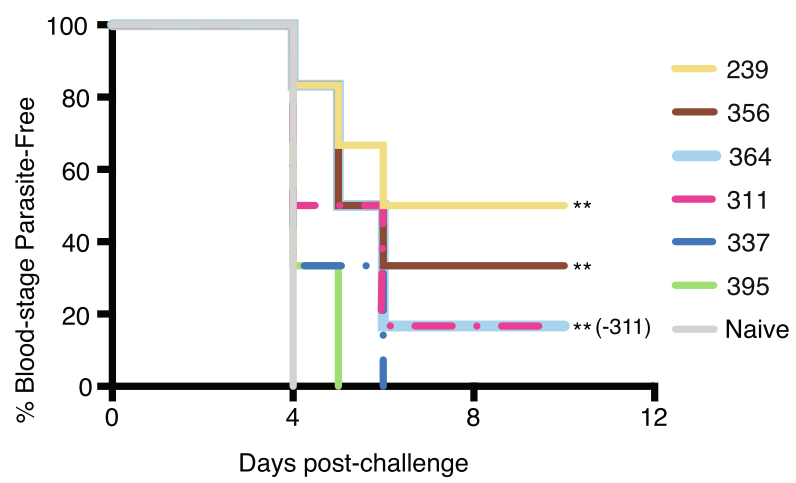

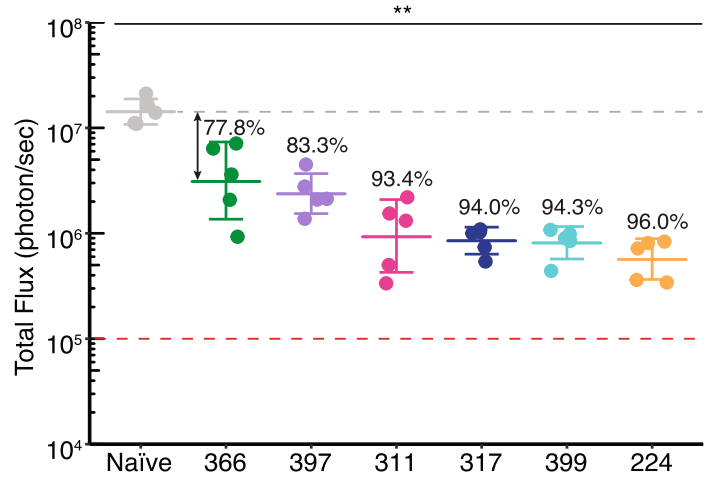
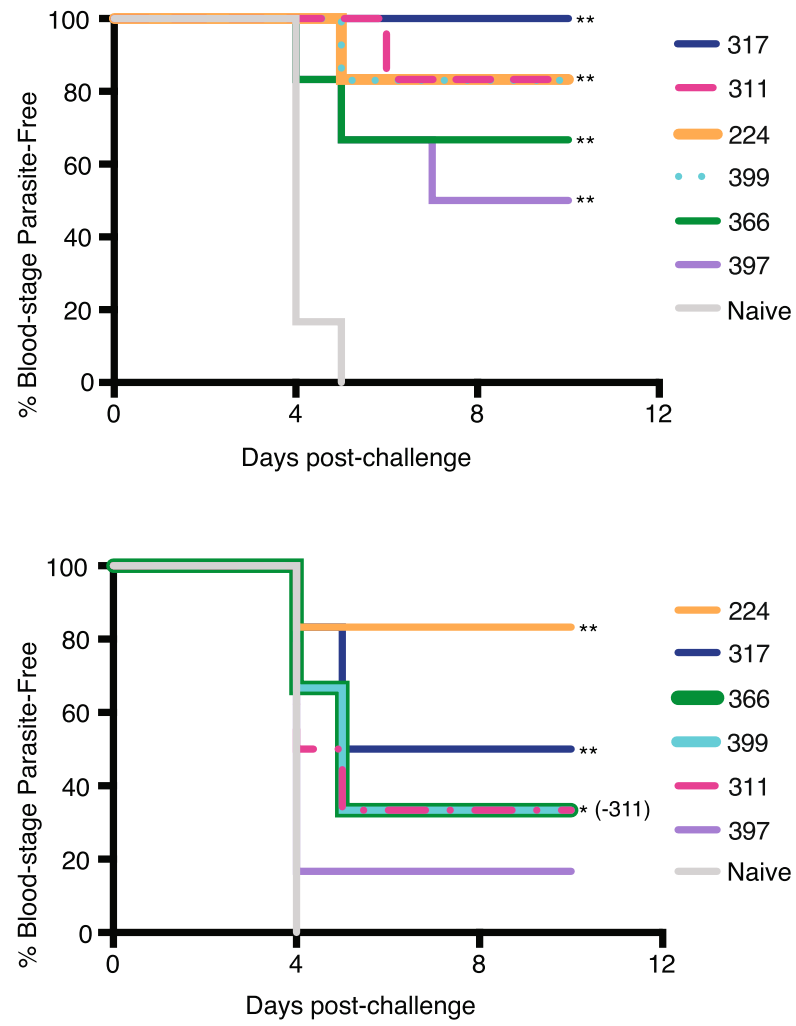

Fig. 1 Assessment of antibody in vivo protection. a Parasite liver burden load was measured by bioluminescence of $P$. berghei sporozoites expressing luciferase-conjugated PfCSP after passive transfer of $100 \mu \mathrm{g}$ of antibody in C57BI/6 mice $(N=5)$. The numbers indicate the percent inhibition of the mean parasite burden relative to that of naïve control mice (i.e., \% inhibition). A two-sided Mann-Whitney U test was used; ${ }^{*} p=0.0159$, and ${ }^{* \star} p=0.0079$. The bar denotes the geometric mean, and error bars denote the SD. Red dashed lines indicate the baseline signal from naïve non-infected mice treated with D-luciferin as established previously ${ }^{32}$. Parasite-free mice after passive immunization with $\mathbf{b} 300 \mu \mathrm{g}$ or $\mathbf{c} 100 \mu \mathrm{g}$ of the indicated antibodies before challenge with bites of infected mosquitoes. A log-rank test was used; in $\mathbf{b}{ }^{\star} p=0.032$ (mAb395), ${ }^{\star \star} p=0.0006$ (mAb239, 356, and 364), 0.0005 (mAb311; second panel, and $\mathrm{mAb317)}, 0.0013$ (mAb399 and 224), 0.0022 (mAb311, first panel), 0.0053 (mAb337), and 0.0089 (mAb366 and 397$) ;$ in (c) ${ }^{*} p=0.019$ (mAb399 and 366 ), ${ }^{\star \star} p=0.0051$ (mAb239, 356, 364, 224, and 317) ( $N=6$ per group). Source data are provided as a Source Data file.

studies $^{33}$ (Fig. 1b, c). At $300 \mu \mathrm{g}$ mAbs, all antibodies from both panels protected at least $50 \%$ of the mice from blood-stage infection compared to the naïve control group $(p<0.05$, log-rank test). Nonetheless, all mAbs, except for those that confer sterile protection, have overlapping confidence intervals (Supplementary Fig. 1), indicating insufficient statistical power $(N=6)$ to distinguish between these mAbs (Fig. 1b). For $100 \mu \mathrm{g} \mathrm{mAbs,}$ only mAb239, 356 and 364 from the first panel, and mAb224, 399,366 , and 317 from the second-panel exhibit protection that was significantly greater than the naïve control mice $(p<0.05$, log-rank test) (Fig. 1c).
Antibody apparent affinity increases with homotypic interactions. Antibody binding affinities were measured using isothermal titration calorimetry (ITC) against both short and long NANP repeat peptides to capture potential increases in apparent affinity due to avidity effects through homotypic Fab-Fab interactions, as observed previously for certain Fabs ${ }^{26,27}$. Despite conventional use of the term "NANP" repeats, our previous studies have consistently shown that anti-NANP antibody epitopes are typically composed of two to three "NPNA" structural motifs ${ }^{27-29}$. Therefore, the binding of all mAbs was measured against $\mathrm{NPNA}_{2}, \mathrm{NPNA}_{4}$, and $\mathrm{NPNA}_{6}$ peptides. 


\begin{tabular}{|c|c|c|c|c|}
\hline $\mathbf{m A b}$ & $\begin{array}{l}K_{\mathrm{d}} \text { to } \\
\text { NPNA }_{2}(\mathrm{nM})\end{array}$ & $\begin{array}{l}K_{\mathrm{d}} \text { to } \\
\text { NPNA }_{4}(\mathrm{nM})\end{array}$ & $\begin{array}{l}K_{\mathrm{d}} \text { to } \\
\text { NPNA }_{6}(\mathrm{nM})\end{array}$ & $\begin{array}{l}\text { Mean } K_{d} \text { fold- } \\
\text { change NPNA } \\
\text { vs NPNA } \\
\end{array}$ \\
\hline 239 & $641 \pm 38$ & $20 \pm 2$ & $10 \pm 1$ & 64 \\
\hline 311 & $152 \pm 11$ & $14 \pm 5$ & $19 \pm 4$ & 8 \\
\hline 337 & $1523 \pm 11$ & $71 \pm 1$ & $59 \pm 14$ & 26 \\
\hline 356 & $8272 \pm 1284$ & $82 \pm 18$ & $30 \pm 6$ & 276 \\
\hline 364 & $47 \pm 2$ & $28 \pm 2$ & $27 \pm 3$ & 2 \\
\hline 395 & $6152 \pm 196$ & $1423 \pm 404$ & $999 \pm 231$ & 6 \\
\hline $\mathbf{m A b}$ & $\begin{array}{l}K_{\mathrm{d}} \text { to } \mathrm{NPNA}_{2} \\
(\mathrm{nM})\end{array}$ & $\begin{array}{l}K_{\mathrm{d}} \text { to } \mathrm{NPNA}_{\mathbf{4}} \\
(\mathrm{nM})\end{array}$ & $\begin{array}{l}K_{\mathrm{d}} \text { to } \mathrm{NPNA}_{6} \\
(\mathrm{nM})\end{array}$ & $\begin{array}{l}\text { Mean } K_{d} \text { fold- } \\
\text { change NPNA }{ }_{4} \\
\text { vs. NPNA }{ }_{6}{ }^{a}\end{array}$ \\
\hline 224 & $14245 \pm 4507$ & $126 \pm 14$ & $141 \pm 8$ & 1 \\
\hline 366 & $1348 \pm 110$ & $1770 \pm 82$ & $479 \pm 17$ & 4 \\
\hline 397 & $2743 \pm 156$ & $255 \pm 10$ & $92 \pm 8$ & 3 \\
\hline 399 & $13345 \pm 2017$ & $5922 \pm 1122$ & $94 \pm 30$ & 63 \\
\hline 317 & $132 \pm 62$ & $70 \pm 12$ & $111 \pm 23$ & 1 \\
\hline
\end{tabular}

The fold-change calculation for these two sets of antibodies is between NPNA $\mathrm{A}_{4}$ and NPNA 6 as it was shown by the crystal structures that their minimal epitopes are $\mathrm{NPNA}_{3}$. Thus, the comparison between NPNA 2 and NPNA 6 would not be appropriate here.

While some Fabs had strong binding (near the nM range) against short NPNA peptides, most Fabs tested here started from low affinity ( $\mu \mathrm{M}$ range) that was dramatically increased against longer NPNA peptides, as indicated by the fold-change of the dissociation constants $\left(K_{\mathrm{d}}\right)$ (Table 1, Supplementary Figs. 2, 3, and Supplementary Table 2). For example, mAb356 recognized $\mathrm{NPNA}_{2}$ with a $\mu \mathrm{M} K_{\mathrm{d}}$ but bound $\mathrm{NPNA}_{6}$ in the $\mathrm{nM}$ range, resulting in an $\sim 300$-fold $K_{\mathrm{d}}$ change (Table 1 ). Overall, most mAbs bound strongly to the longer NPNA peptides, except for mAb395 and 366, whose affinity remained well within the $\mu \mathrm{M}$ range (Table 1). Multiple copies of Fab311, with an 8-fold mean $K_{\mathrm{d}}$ change between $\mathrm{NPNA}_{2}$ and $\mathrm{NPNA}_{6}$, simultaneously recognized rsCSP (NPDP/NVDP/NANP repeat ratio of $1 / 3 / 19$ instead of $1 / 4 / 38$ for the P. falciparum 3D7 strain) and exhibited homotypic inter-Fab contacts ${ }^{27}$. Therefore, mAbs 239, 337, 356, and 399 with higher fold-changes should also exhibit Fab-Fab interactions on binding multiple epitopes on the CSP NANP repeat region (Table 1 ).

The presence of homotypic Fab-Fab interactions in mAbs 239 and 399 was validated in the crystal structures of Fab239-NPNA and Fab399-NPNA 6 complexes (Fig. 2, Supplementary Table 3). Two copies of Fab239 form side-to-side inter-Fab contacts, which are mediated mostly by the heavy chain (Fig. 2a-c). These Fab-Fab interactions are asymmetric where complementaritydetermining region (CDR) $\mathrm{H} 1$ and $\mathrm{H} 3$ in one Fab interact mainly with CDR H2 and L3 in the adjacent Fab (Fig. 2b, c, g), which is strikingly similar to what is observed in the cryo-EM structure of Fab311 with rsCSP $^{27}$. The interaction of ${ }^{\mathrm{H}} \mathrm{Asp}^{31}$ (Asp residue 31 from the heavy chain) from CDR $\mathrm{H} 1$ with ${ }^{\mathrm{H}_{\mathrm{Glu}}}{ }^{64}$ from CDR H2 is also conserved in the Fab-Fab interfaces of Fabs 239 and 311 (Fig. 2c). Therefore, these similarities suggest that Fab239 may also be capable of forming a long-range spiral structure, in which multiple copies of the Fab simultaneously bind to $\mathrm{rsCSP}^{27}$. Fab399 exhibits a different type of Fab-Fab interaction with a head-to-head configuration, resembling that of Fab1210 26 (Fig. 2d, e), but the inter-Fab contacts here are perfectly symmetric, more extensive, and mediated almost entirely by the heavy chain. ${ }^{\mathrm{H}} \mathrm{Asp}^{31}$ in CDR $\mathrm{H} 1$ now forms a different network

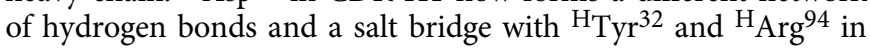
the adjacent Fab (Fig. 2f, g). Unlike Fabs 239 or 311, Fab399 is not likely to form a similar spiral conformation with $\mathrm{rsCSP}^{27}$ because such a structure would not accommodate the symmetric, head-tohead, inter-Fab interactions. Despite the different CDR contributions to the homotypic interactions of Fabs 239 and 399, both antibodies similarly use CDR $\mathrm{H} 1, \mathrm{H} 2, \mathrm{H} 3$, and $\mathrm{L} 3$ for interaction with the NANP peptide (Supplementary Fig. 4).

Antibody in vivo protection correlates with Fab apparent affinity. To approximate the binding affinity and kinetics of these Fabs to full-length CSP, bio-layer interferometry (BLI) was performed using rsCSP (Table 2, Supplementary Fig. 5). The overall $K_{\mathrm{d}}$ values with BLI were similar to those obtained from ITC, with mAbs 395 and 366 displaying the lowest affinity well within the $\mu \mathrm{M}$ range (Tables 1,2 ). Most $\mathrm{mAbs}$ with high apparent affinity exhibited potent functional activity in the mouse model of parasite liver burden load, except for mAbs 337 and 397, which performed worse than expected. The low-affinity mAbs 366 and 395 performed less well, as anticipated (Table 2 ). Although only 11 antibodies were examined in this study, the percent inhibition of parasite load, normalized across the two antibody panels, exhibited a correlation with Fab apparent affinity to $\mathrm{NPNA}_{6}$ peptide by ITC and to rsCSP by BLI with $\mathrm{R}^{2}$ of 0.8435 and 0.9257 , respectively (Table 2 and Fig. 3). For cross-validation of the linear regression, bootstrapping was performed to generate 1000 models with an average $R^{2}\left(R^{2}\right.$-boot) of 0.6819 and 0.7763 for apparent affinity against both $\mathrm{NPNA}_{6}$ peptide and rsCSP vs. normalized percent inhibition, respectively, which was lower than the $R^{2}$ of the original models but still suggested a linear correlation (Fig. 3). In contrast, the affinity against the short $\mathrm{NPNA}_{2}$ peptide did not correlate with the inhibition of parasite liver burden load, nor did the mean $K_{\mathrm{d}}$ fold-change from ITC (Supplementary Fig. 6 and Table 1). All antibodies had equivalent association rate constants $\left(k_{\text {on }}\right)$ in the order of $10^{4} \mathrm{M}^{-1} \mathrm{~s}^{-1}$ that did not correlate with in vivo protection from the liver burden assay (Table 2 and Fig. 3). On the other hand, the most potent blocking antibodies displayed comparable dissociation rate constants $\left(k_{\text {off }}\right)$ in the range of $10^{-3} \mathrm{~s}^{-1}$, whereas the less potent mAbs 366 and 395 had faster $k_{\text {off }}$ of $\sim 10^{-2}$ and $\sim 10^{-1} \mathrm{~s}^{-1}$, respectively (Table 2). Thus, the $k_{\text {off }}$ component of the $K_{\mathrm{d}}$ correlated well with the normalized percent inhibition from the liver burden assay $\left(R^{2}=0.9230, R^{2}\right.$ boot $=0.7633$ ) (Table 2 and Fig. 3 ). Antibody thermal stability $\left(T_{\mathrm{m}}\right)$ was also examined but showed no correlation with antibody activity as measured by liver burden load (Table 2, and Supplementary Fig. 6). Thus, the outlier mAbs 337 and 397 with high affinity but poor protection could result from poor pharmacokinetics, e.g., durability and clearance in mice, aggregation in mouse serum, or cross-reactivity with mouse antigens etc.

Crystal structures reveal conserved interaction motifs. To gain a better understanding of the molecular recognition of the antibodies to the NANP repeats and what structural features might relate to low binding affinity and poor protection, we cocrystalized Fabs 239, 356, 364, 395, 224, 399, and 366 with repeat peptides $\left(\mathrm{NPNA}_{2,3,4}\right.$, or 6 ), and compared with our previous crystal structures of Fabs 311, 317, and 39728,29. Except for Fab395, the epitopes of the IGHV3-33 Fabs share a similar conformation that includes a type I $\beta$-turn and an Asn pseudo $33_{10}$ turn formed by the first and the second NPNA motifs, respectively (Fig. 4a, Supplementary Table 4). Although the Fab395 epitope is shorter $\left({ }^{1} \mathrm{NPNANP}{ }^{6}\right)$, the first NPNA motif also adopts a type I $\beta$-turn (Fig. 4b). The NANP peptides in the IGHV3-33 Fabs interact mainly with the heavy chain as indicated by its dominant contribution to the buried surface area (BSA); the light-chain interactions are mostly mediated through CDR L3 (Fig. 4a, b, and Supplementary Table 5). The IGHV3-33 Fabs all have similar conformations of CDR $\mathrm{H} 1$ and $\mathrm{H} 2$, which contribute 
a Fab239-NPNA 4 complex

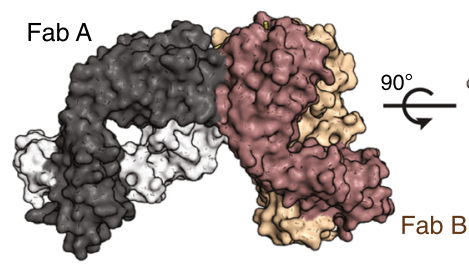

C
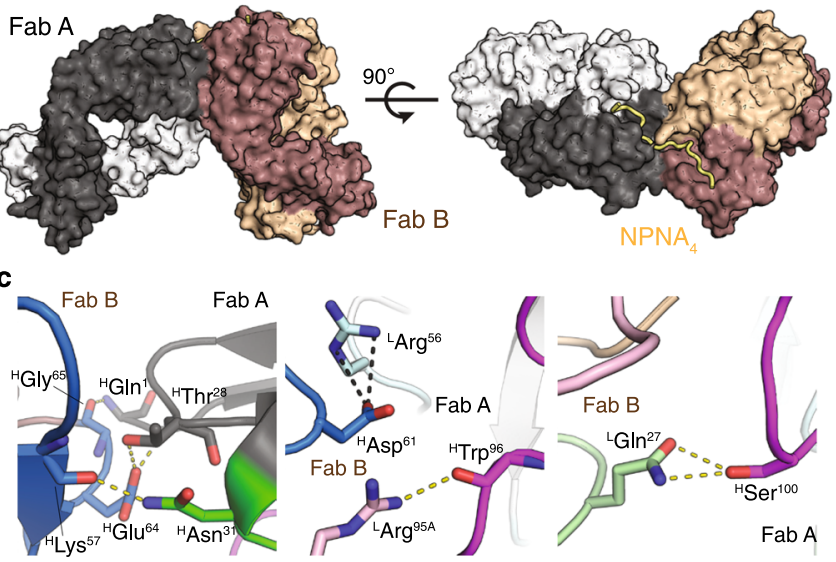

b

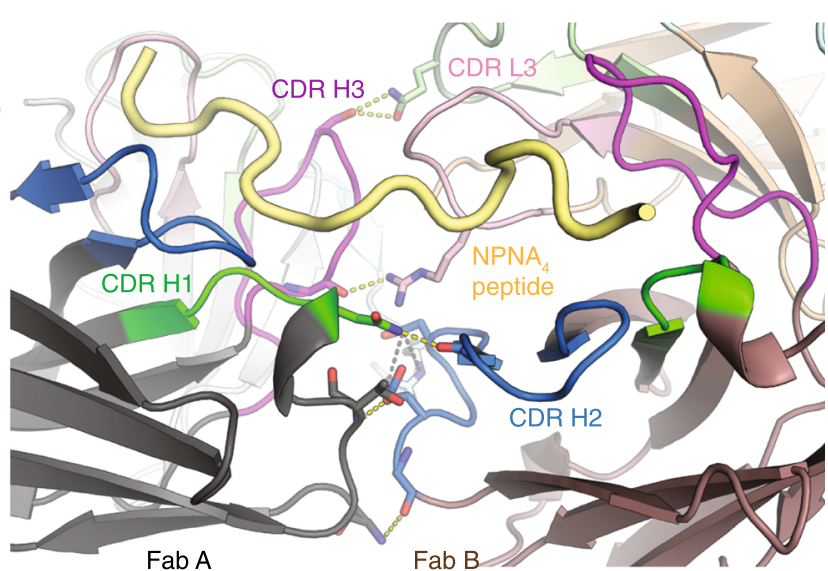

d Fab399-NPNA 6 complex e

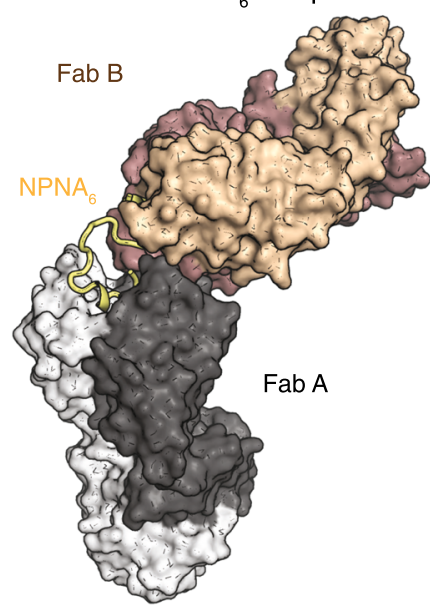

-
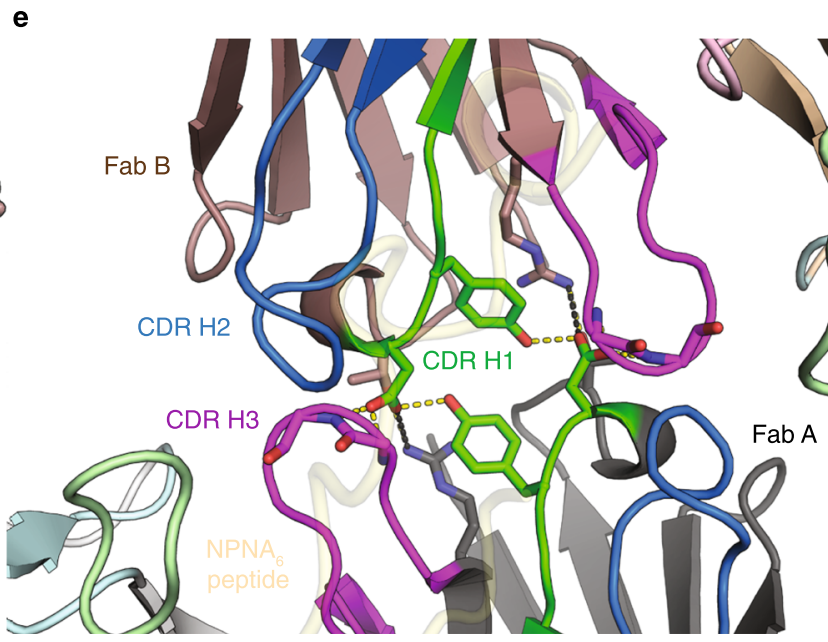

$\mathbf{f}$

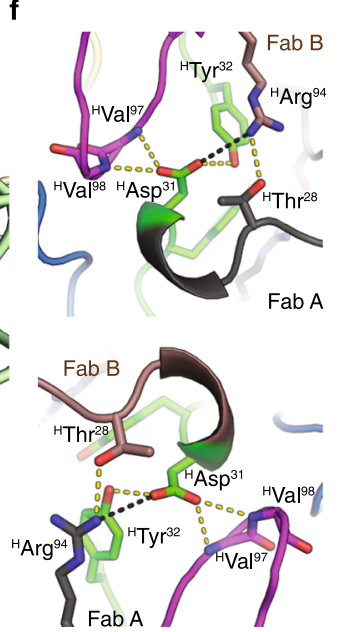

g

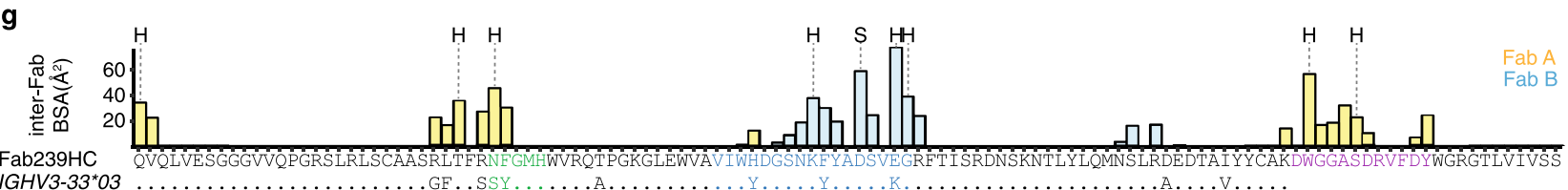

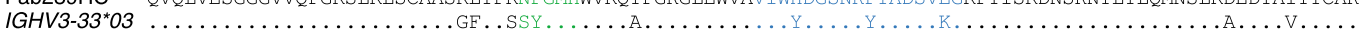

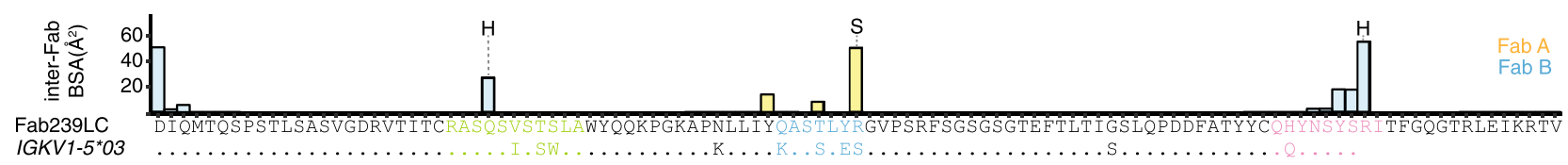

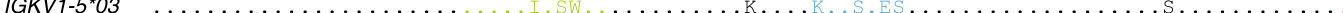

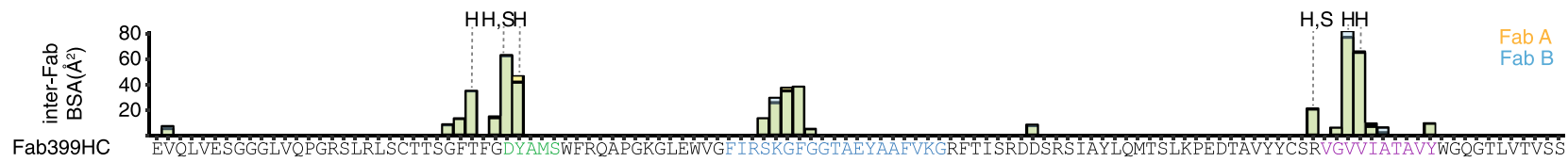

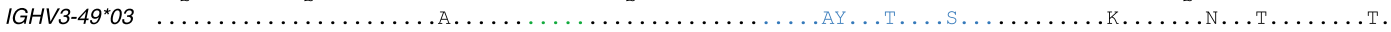

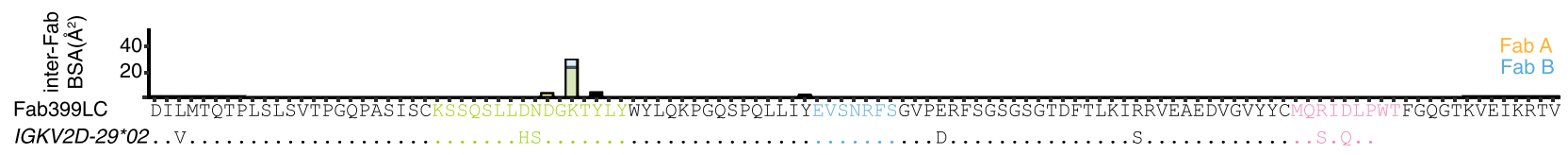

to conserved interactions with their epitopes, with Fab395 having a unique disulfide bond between ${ }^{\mathrm{H}} \mathrm{Cys}^{29}$ in the framework region and ${ }^{\mathrm{H}} \mathrm{Cys}^{32}$ in CDR H1 (Kabat nomenclature) (Fig. 4c, and Supplementary Table 6). Notably, the main chain of residues in CDR $\mathrm{H} 1$ form a conserved hydrogen bonding network with the NANP peptide. Conservation of van der Waals interactions are mediated by aromatic residues $32 / 33$ in CDR H1, and 52 and 58 in CDR H2 (Fig. 4c, and Supplementary Table 6). While Tyr in position $32 / 33$ and 58 can evolve to other aromatic residues, ${ }^{\mathrm{H}} \mathrm{Trp}^{52}$ is strictly conserved in our antibody panel. Two of these residues, 52 and 58, interact specifically with Pro in the Asn pseudo $3_{10}$ turn and type I $\beta$-turn respectively (Fig. $4 \mathrm{c}$, and Supplementary Table 6). Although Fab395 displays a seemingly different epitope compared to other IGHV3-33 Fabs in this panel, 
Fig. 2 Homotypic Fab-Fab interactions in Fab239- and Fab399-peptide complexes. a Two copies of Fab239 (Fab A, black: heavy chain, white: light chain. Fab B, dark brown: heavy chain, tan: light chain) in the crystal structure are shown as surfaces with the NPNA 4 peptide represented as a yellow tube. b, $\mathbf{c}$ Interactions between two Fabs ( $\mathrm{A}$ and $\mathrm{B}$ ) that simultaneously recognize an NPNA 4 peptide. Hydrogen bonds (yellow dashes) and salt bridges (black dashes) are highlighted. The Fabs are shown as cartoon representations with the side chains of interacting residues represented as sticks. CDRs as defined by Kabat are colored green, blue, magenta, light green, light cyan, and pink for CDR H1, H2, H3, L1, L2, and L3, respectively. d Surface representation of homotypic, head-to-head interactions of two copies of Fab399 in the crystal structure with NPNA 6 peptide (yellow tube). e, $\mathbf{f}$ Contacts between two Fabs that simultaneously recognize the NPNA 6 peptide (transparent yellow tube). All coloring schemes and representations are as for the Fab239-NPNA 4 complex. $\mathbf{g}$ Individual residue contributions to the BSA of inter-Fab interactions are shown in a bar plot for the heavy and light chains of Fab239 and Fab399. The yellow and blue bars indicate the BSA on Fab A and Fab B (defined as in previous panels), respectively, while the green bars show the overlap of both. The CDRs are colored as in previous panels. Additionally, the alignment between the Fab heavy/light chain sequences and germline IGHV and IGKV gene sequences is shown to display somatically mutated residues. " $\mathrm{H}$ " and " $\mathrm{S}$ " mark residues that are engaged in hydrogen bonds and salt bridges, respectively. Antibody amino acid residues are labeled with a superscript " $\mathrm{H}$ " for heavy chain and " $\mathrm{L}$ " for light chain. Residue numbers are also shown as superscripts.

the type I $\beta$-turn in the Fab395 epitope is positioned in the pocket where the Asn pseudo 310 turn is accommodated in other Fabs, such as Fab311 (Fig. 4b). The Pro in the type I $\beta$-turn now interacts with ${ }^{\mathrm{H}} \mathrm{Trp}^{52}$ and the following Pro contacts ${ }^{\mathrm{L}} \mathrm{Tyr}^{98}$. The presence of the bulky ${ }^{\mathrm{L}} \mathrm{Tyr}^{94}$ in CDR L3 of Fab395 may prevent binding of the repeat peptide to the usual pocket for the type I $\beta$ turn as in other IGHV3-33 Fabs (Fig. 4b). Consequently, the binding pocket of Fab395 is considerably smaller and may contribute to its low binding affinity and poor protection.

The crystal structures of Fab224 and Fab399, which are derived from the same variable heavy chain IGHV3-49 gene, also display similarities in their recognition of the NANP repeats (Fig. 5a, b, and Supplementary Table 3 and 4). The epitopes are mainly composed of three NPNA motifs (Fab399 only has NPN in the last motif) with the first two NPNA motifs adopting a type I $\beta$ turn and the last motif exhibiting a type I $\beta$-turn in Fab224 and an Asn pseudo $3_{10}$ turn in Fab399 (Fig. 5a, b). The interactions of Fabs 224 and 399 with the NANP peptides are mediated mainly through the heavy chain (Fig. 5a, b and Supplementary Table 5), which resembles those observed in the IGHV3-33 antibodies (Fig. 4a, b). The binding pocket formed by the IGHV3-49 heavy chains in these two Fabs both recognize the type I $\beta$-turn through nearly identical molecular interactions, including a $\mathrm{CH}-\pi$ interaction between the germline-encoded ${ }^{\mathrm{H}} \mathrm{Phe}^{50}$ and Pro in the NPNA repeat (Fig. 5a, b). Other shared interactions involve hydrogen bonds and van der Waals interactions mediated by ${ }^{\mathrm{H}} \mathrm{Arg}^{52}$ and ${ }^{\mathrm{H}} \mathrm{Tyr}^{53}$, or the somatically mutated ${ }^{\mathrm{H}} \mathrm{Phe}^{53}$ in Fab399, and hydrogen bonds from the CDR H3 backbone to the NANP peptide (Fig. 5a, b). Fabs 224 and 399 both have a Trp in CDR L3 ( ${ }^{\mathrm{L}} \operatorname{Trp}^{96}$ ) that hydrogen bonds with the backbone of the Ala preceding the conserved NPNA type I $\beta$-turn (Fig. 5a, b). However, their light chains are derived from different germline genes (Supplementary Table 5). The Fab399 epitope also shares striking similarities with the epitope in a recently published, IGHV3-49-encoded Fab4493 crystal structure with the junctional peptide, GNPDPNANPN ${ }^{24}$. The PDPNANPN core of the Fab4439 epitope displays a nearly identical conformation to the ANPNANPN residues in the Fab399 epitope, and both epitopes make similar interactions with ${ }^{\mathrm{H}} \mathrm{Phe}^{50}, \mathrm{H}_{\mathrm{Arg}}{ }^{52}$, and ${ }^{\mathrm{L}} \mathrm{Trp}{ }^{96}$ in the antibody paratopes ${ }^{24}$.

We also determined the structure of Fab366 derived from the IGHV1-2 gene for which a structure has not been reported previously for anti-NANP antibodies. Its paratope is composed from CDR H1, H3, L1, and L3 without contribution from CDR $\mathrm{H} 2$, which is so dominant in Fabs from the IGHV3-33 and IGHV3-49 families (Fig. 5c, and Supplementary Table 4). The epitope (from $\mathrm{N}$ - to $\mathrm{C}$-terminus) spans a shallow binding groove from light to heavy chain and makes extensive hydrogen bonds with $\mathrm{Fab}$ residues. One notable interaction is between ${ }^{\mathrm{L}} \operatorname{Trp}{ }^{96}$ and Asn 5 in the peptide, which is similar to that observed in the Fab317- and Fab397-peptide complexes ${ }^{28,29}$ (Fig. 5c).
Interestingly, the Fab366-bound peptide does not contain any secondary structural motifs (i.e., type I $\beta$-turn and Asn pseudo $3_{10}$ turn), but adopts a more extended conformation. In fact, the observation of a shallow Fab paratope recognizing extended NANP repeats resembles the epitope of Fab1450, which also has low affinity and poor in vivo protection ${ }^{26}$ (Fig. 5c). Despite the extensive hydrogen-bonding network and BSA contribution within the range of the other Fabs analyzed here (Fig. $5 c$, and Supplementary Table 5), Fab366 has a fast $k_{\text {off, }}$, hence low affinity, (Table 2), which seems attributable to a shallow binding groove, consistent with its similarity to Fab1450.

Another possible structural correlate of high affinity and better protection is the presence of local secondary conformations in the NANP epitopes, which are the type I $\beta$-turn and Asn pseudo $3_{10}$ turn with a hydrogen bond between the side chain of Asn (residue i) and backbone nitrogen of Asn (residue $\mathrm{i}+2$ ) (Fig. 6). The peptides in potent and high affinity IGHV3-33 antibodies (mAbs 239, 311, 356, and 364) contain both an NPNA type I $\beta$-turn and Asn pseudo $3_{10}$ turn (Table 2, and Fig. 6). Interestingly, the spiral rsCSP contains these two turns as a repeating unit, as observed in the cryo-EM structure with Fab31127. The epitopes of protective mAbs 317, 224, and 399 consist of up to three type I $\beta$-turns, but also display backbone-to-backbone $\mathrm{H}$-bonds, resulting in a more compact conformation, compared to those with the IGKV3-33 antibodies, and also higher apparent affinity to rsCSP (Table 2, and Fig. 6). The mAb397 epitope exhibits both type I $\beta$ - and Asn pseudo $3_{10}$ turns (Fig. 6), which correspond to the structural repeat in the long-range, curved conformation when multiple copies Fab397 bind to rsCSP ${ }^{29}$. However, other factors, such as antibody pharmacokinetics, may account for the poor protection despite high binding affinity of mAb397. In contrast, the low affinity and weakly protective mAb395 has a shorter epitope that contains one type I $\beta$-turn, whereas the extended epitope of mAb366 is stabilized by only one $\mathrm{H}$-bond between the side chain of Asn3 (i) and backbone oxygen of Asn5 $(i+2)$, leading to a turn that is wider and more open than a type I $\beta$-turn (Fig. 6). Consequently, the extended epitopes of mAb366 when repeated in CSP may not be capable of adopting either the long-range curved or spiral conformations that are observed in mAbs 397 and 311 , respectively.

\section{Discussion}

This study strengthens and extends previous observations of recurring features of antibody recognition to the NANP repeats that pertain to their functional activity. Notably, we explored antibodies that were encoded by different sets of IGHV genes, the mechanisms by which different antibodies can achieve high Fab binding affinity, and the consequences for functional protection in liver burden and parasitemia models of infection. We also identified two additional antibodies, mAb239 (IGHV3-33) and mAb399 (IGHV3-49), that display homotypic inter-Fab contacts, 


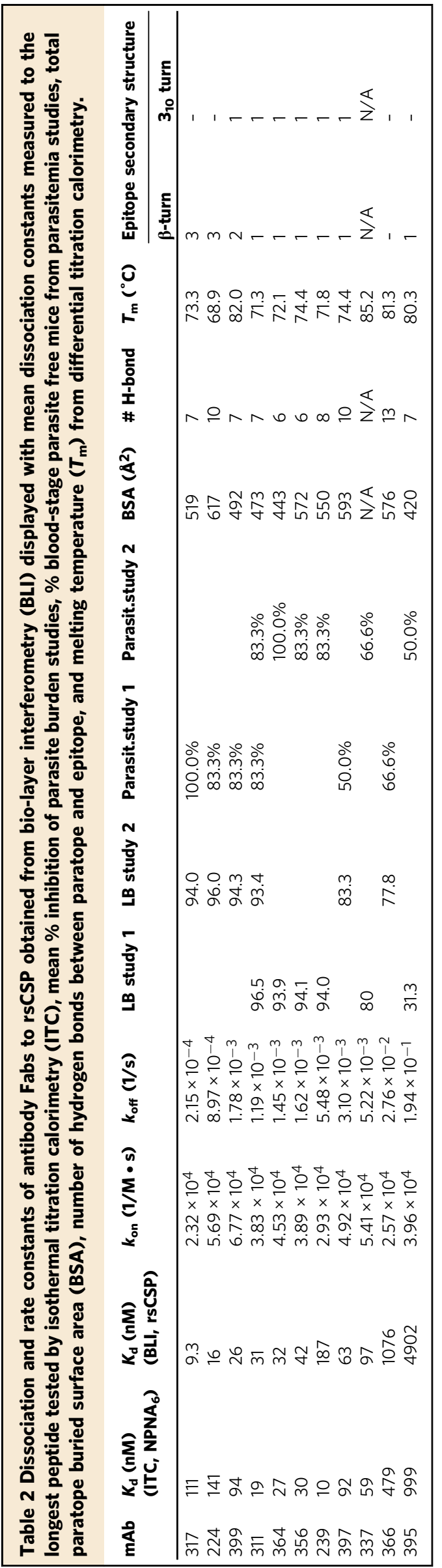

while simultaneously recognizing NANP repeat epitopes, as observed previously in mAbs 311 and 1210 (both from IGHV3$33)^{26,27}$. Fabs 239 and 311 exhibit an asymmetric, side-to-side Fab-Fab interaction with propensity to form a supramolecular, extended spiral structure with rsCSP, whereas Fab399 shows symmetric inter-Fab contacts with a head-to-head configuration that may not be consistent with forming the same type of spiral architecture, and may be more similar to that observed in Fab1210 26 . We note that some of the key residues involved in homotypic Fab-Fab interactions are already present in the germline genes for both Fabs 239 and 399 (Fig. 2g). Indeed, most residues involved in inter-Fab contacts in Fab399 are germlineencoded, such as ${ }^{\mathrm{H}} \mathrm{Thr}^{28}, \mathrm{H}_{\mathrm{Asp}}{ }^{31}, \mathrm{H}_{\mathrm{Tyr}}{ }^{32}$, and ${ }^{\mathrm{H}} \mathrm{Arg}^{94}$ (Fig. 2f, g). Therefore, these two antibodies may exhibit a propensity for inter-Fab contacts, even prior to somatic hypermutation (SHM). A similar observation is found in rituximab (RTX) against CD20, a completely unrelated therapeutic antibody ${ }^{34}$. Two copies of the Fab display homotypic interactions upon binding to their epitope on the CD20 dimer, resulting in a cross-linked circular supraassembly of three RTX IgGs and three CD20 dimers ${ }^{34}$. Intriguingly, all residues involved in the RTX Fab-Fab interactions are also germline-encoded ${ }^{34}$.

The question remains whether the selection and subsequent maturation of these inter-Fab contacts impact antibody maturation or functional activity. The prevalence of an immunogenic repeat region in CSPs across different Plasmodium species ${ }^{10}$, albeit with distinct repeating sequence motifs, suggests that this region may act as an immune decoy ${ }^{35}$ by favoring the selection of antibodies with Fab-Fab interactions. A study of mAbs produced by immunization with $P f$ sporozoites indicates that clonal selection of higher-affinity, germline B-cell receptors (BCRs), rather than efficient SHM, seems to drive anti-NANP responses ${ }^{36}$. These germline BCRs may then represent the precursors of anti-NANP mAbs that display inter-Fab contacts. Consequently, it was hypothesized that high-avidity crosslinking of BCRs from homotypic contacts may signal B-cells to exit the germinal center either prematurely or with limited rounds of somatic mutation, especially in the antibody-antigen interface, and perhaps disfavor the formation of long-lived plasma cells, which are responsible for generating high amount of circulating antibodies as immediate responses to sporozoite invasion ${ }^{37}$. The increasing number of observations of homotypic Fab-Fab interactions in antiNANP antibodies here and in previous studies ${ }^{26,27}$, and recently in an antibody targeting CSP on a different Plasmodium species, $P$. berghei ${ }^{38}$, support the above hypothesis. Fab-Fab interactions boost apparent antibody affinity through avidity effects and are also shared features among protective anti-NANP mAbs. However, such contacts may not be beneficial for the formation of immune memory and antibody potency and thus durable antibody responses, and further suggests that short NANP repeat based immunogens to prevent or modulate homotypic contacts might have some advantages to consider in the design of next-generation CSP-based malaria vaccines. Such immunogens date back to the 1980 s when short NANP repeats $\left[(\mathrm{NANP})_{3}\right.$ ] conjugated to tetanus toxoid were tested in a human trial and induced strong anti-NANP antibody responses ${ }^{39}$, but no booster effect due to the lack of T-cell epitopes e.g. from other non-repeat domains ${ }^{40}$. Interestingly, recent immunization studies with a truncated CSP with only 9 NANP repeats induced lower BCR signaling in NANP-repeat-specific Bcells, stronger responses to $\mathrm{N}$ - and C-term epitopes, and protected more mice against mosquito bite challenge as compared to immunization with a longer CSP containing 27 NANP repeats ${ }^{41}$. Whether this approach will lead to more durable antibody responses and robust immune memory remains to be determined.

The crystal structures reported here of additional antibodies derived from the IGHV3-33 gene further emphasize the role of the conserved $\operatorname{Trp}^{52}$ in CDR H2 for interaction with Pro in the 
a Apparent affinity to $\mathrm{NANP}_{6}$ (ITC) vs. Normalized \% inhibition

b Apparent affinity to rsCSP (Octet) vs. Normalized \% inhibition
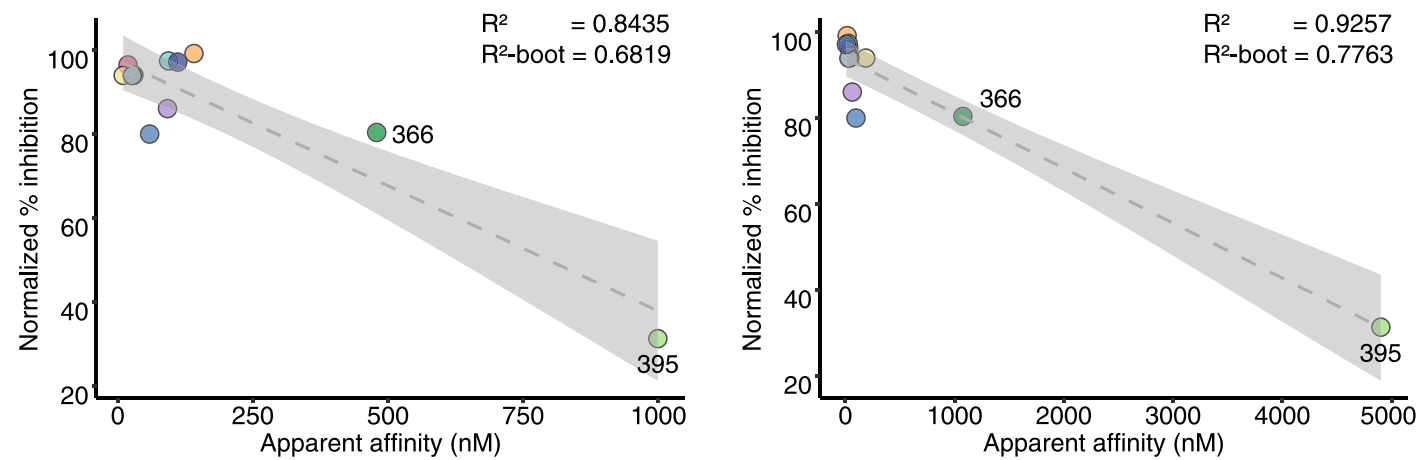

c $\mathrm{k}_{\text {on }}$ to rsCSP (Octet) vs. Normalized \% inhibition

d $\mathrm{k}_{\text {off }}$ to rSCSP (Octet) vs. Normalized \% inhibition
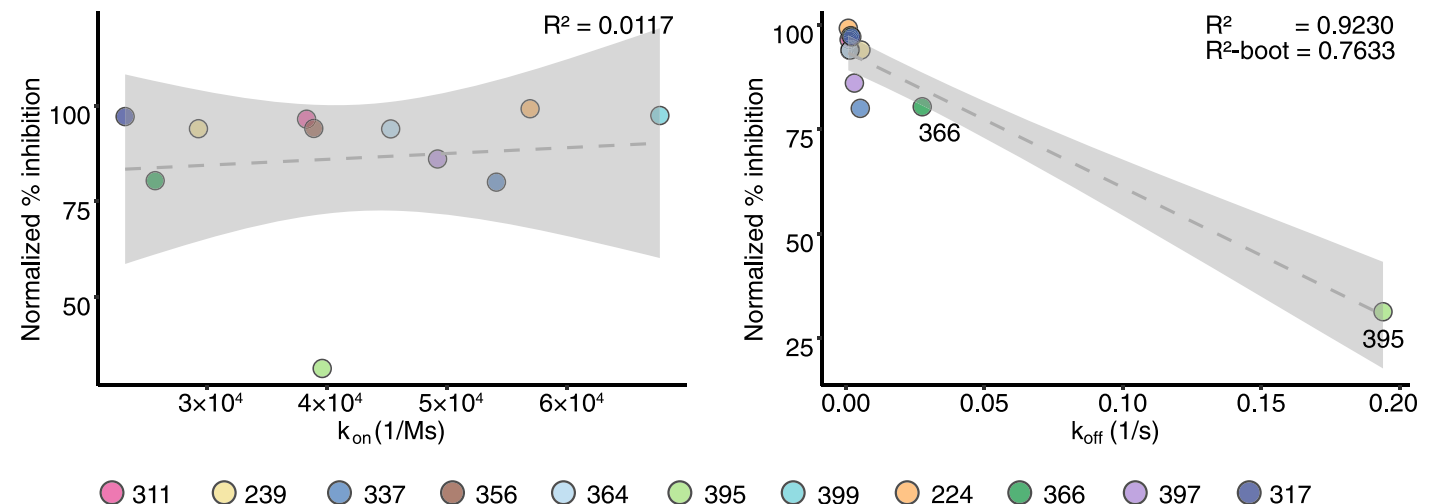

Fig. 3 Correlation of apparent affinity and dissociation constants with normalized parasite burden. The linear regression graph plots the \% inhibition of the parasite liver burden load, normalized across the two mAb panels, against: a Fab apparent affinity as the dissociation constant $\left(K_{d}\right)$ measured to the $\mathrm{NPNA}_{6}$ peptide using isothermal titration calorimetry; $(\mathbf{b}) K_{\mathrm{d}}(\mathbf{c})$ association rate constant $\left(k_{\mathrm{on}}\right)$, and $(\mathbf{d})$ dissociation rate constant $\left(k_{\text {off }}\right)$ measured against rsCSP using bio-layer interferometry. The dashed line indicates the fitted linear regression model with $95 \%$ confidence interval shaded in grey. The $R^{2}$ value for each model is displayed, together with the average $R^{2}$ from 1000 models from bootstrapping ( $R^{2}$-boot). Data points for each $\mathrm{mAb}$ are colored as shown. Source data are provided as a Source Data file.

NANP repeats (Fig. 4). Alanine substitution not only of ${ }^{\mathrm{H}} \operatorname{Trp}^{52}$, but also ${ }^{\mathrm{L}} \operatorname{Trp}^{32}$, and ${ }^{{ }^{H}} \operatorname{Trp}^{33}$ in mAbs 311, 317, and 397, disrupts antibody binding considerably ${ }^{29}$. The convergent usage of Trp is also highlighted by the interaction between ${ }^{\mathrm{L}} \operatorname{Trp}{ }^{96}$ encoded by the light chain $J$ gene in mAb366 with Asn in the NANP repeats (Fig. 5c), whereas ${ }^{\mathrm{L}} \operatorname{Trp}^{96}$ in Fab224 and 399 hydrogen bonds with the alanine backbone in the NPNA peptide (Fig. 5a, b). We also observed that $\mathrm{HPhe}^{50}$ was present in two antibodies from the IGHV3-49 germline for interaction with Pro in the conserved NPNA type I $\beta$-turn, similar to the Trp in IGHV3-33 mAbs (Fig. 5a, b). Other aromatic residues, such as His and Tyr, can form van der Waals interactions with the peptide as seen in CDR $\mathrm{H} 2$ of IGHV3-33 antibodies (Fig. 4c). The high prevalence of such interactions between Fab aromatic residues and the NANP peptide is summarized for anti-NANP and anti-junction mAbs in Fig. 7. A recent cryo-EM structure of mouse mAb3D11 against the repeat region of $P$. berghei CSP reveals that $3 \mathrm{D} 11$ uses eight aromatic residues to form an aromatic cage for antigen recognition, with a germline-encoded Tyr from the light-chain playing a key role ${ }^{38}$. These structural insights suggest that the NANP repeats in PfCSP prime the human immune system to select antibodies from germline genes with well-positioned aromatic residues for the initial encounter. These favorable, dominant interactions with germline-encoded aromatic residues may limit SHM and represent another hurdle that the NANP repeats pose for eliciting durable and more potent human antibody responses.

A recent study has shown that antibody affinity to NANP peptides correlates with inhibition of the parasite's traversal of hepatocytes in vitro and also that antibodies with high affinity to NANP, rather than the other motifs (e.g., NPDP, or NVDP) in the junctional region, exhibit a high level of protection in the mouse model of parasitemia ${ }^{24}$. Here, we also demonstrate a correlation between apparent affinity, as measured against both NANP repeats and rsCSP, and in vivo protection in the liver burden assay (Fig. 3, and Tables 1 and 2). The parasitemia data also follow this trend, but are not a large enough dataset to assign statistical significance. Other structural and biophysical features, which include total paratope BSA, number of hydrogen bonds between paratope and epitope, and antibody melting temperature, do not appear to correlate with in vivo protection (Table 2 and Supplementary Fig. 6). Perhaps not surprisingly, the anti-NANP antibodies share a similar $k_{\text {on }}$, but can differ substantially in their $k_{\text {off }}$, which explains the lower affinities observed for mAbs 366 and 395 (Table 2). The $k_{\text {off }}$ then dictates the linear correlation with normalized percent inhibition of parasite burden (Fig. 3). However, the caveat for the current analysis is that only two antibodies, mAbs 366 and 395, in this study have low affinity and poor in vivo protection, and, hence, these two data points tend to dominate the regression models. As mAbs analyzed in this study were initially screened based on their avidity on ELISA, lowaffinity antibodies are then likely to be underrepresented. To reduce model bias, we also performed bootstrapping to generate 1000 models for each analysis and observed lower average $R^{2}$ values $\left(R^{2}\right.$-boot), but that still indicate correlation with in vivo protection (Fig. 3). Despite these limitations, our results should serve as an important platform for the development and 
a Fab311, 239, 356, and 364

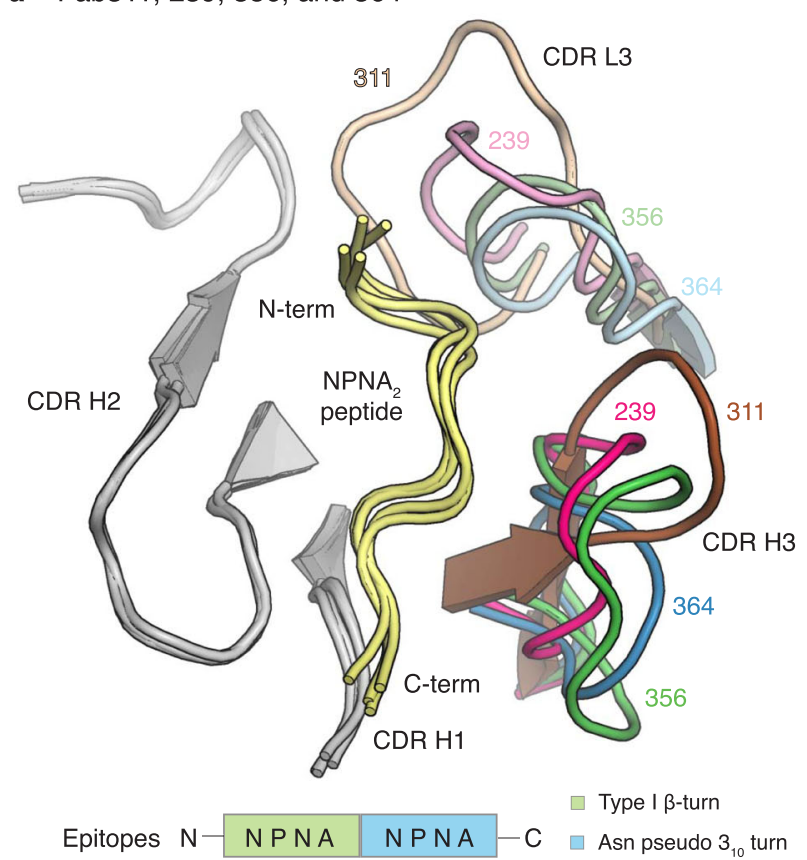

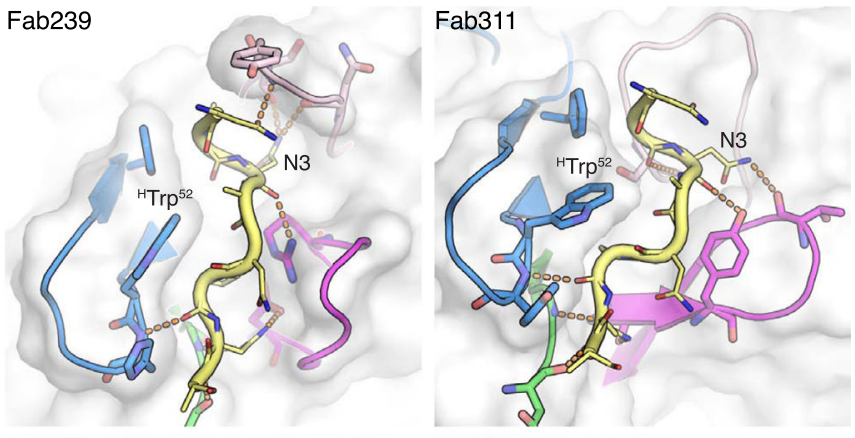
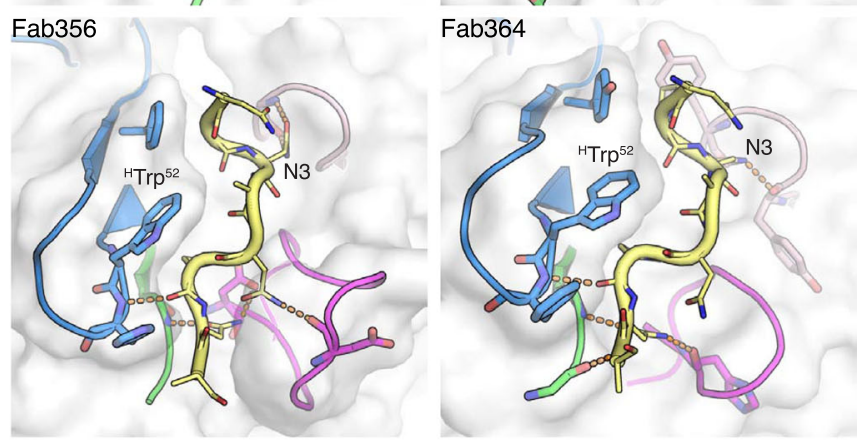

b Fab311 vs. Fab395

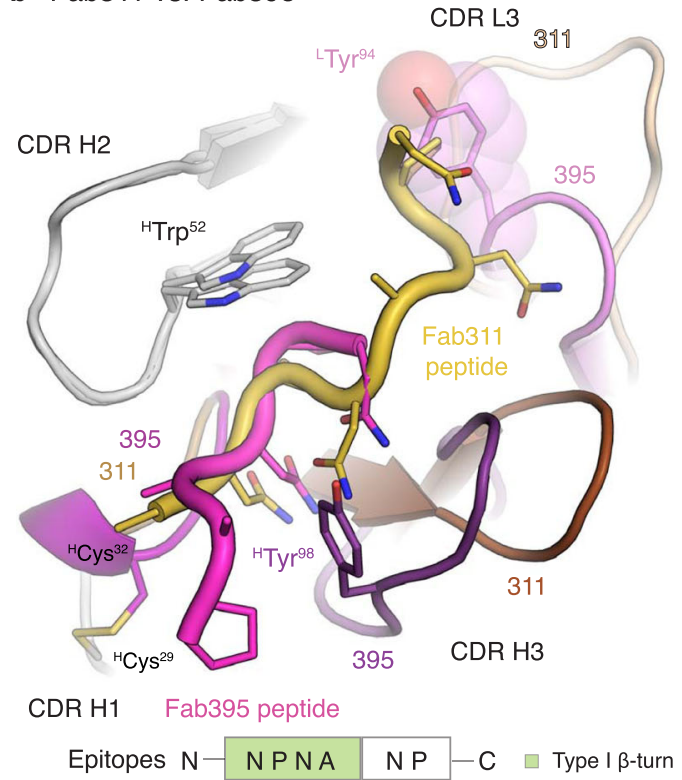

c Conserved molecular interactions

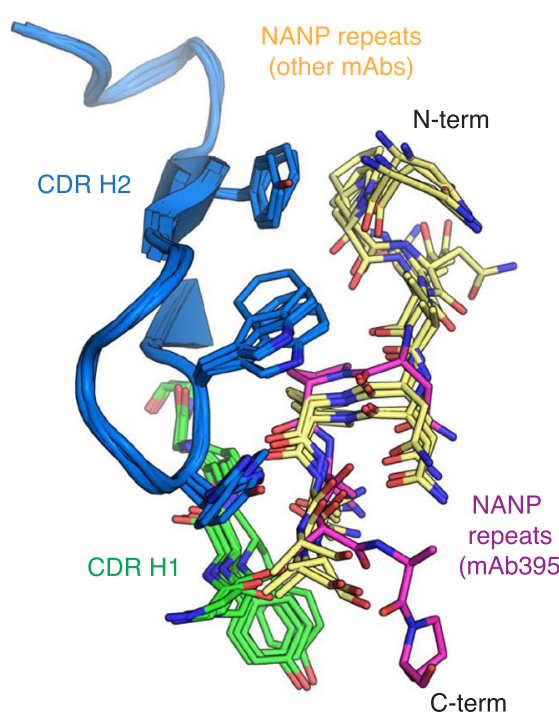

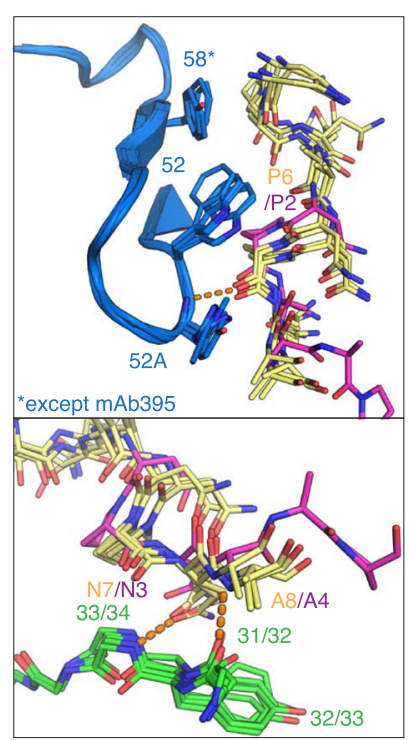

Fig. 4 Crystal structure of IGHV3-33 Fabs. a Structures of Fab239, 311, 356, and 364 in complex with NPNA 2 peptide (yellow) are shown in cartoon representation and aligned based on CDR H2. Only the CDRs involving in peptide binding are shown with CDR H1 and $\mathrm{H} 2$ colored in grey and CDR $\mathrm{H} 3$ and L3 colored for the different antibodies as indicated. A schematic of the epitope structural motifs is also indicated below. Close-up views of the paratopes are also displayed with the Fabs as cartoons embedded in their surface representation. $\mathrm{CDR} \mathrm{H1}, \mathrm{H2}$, H3, and L3 are colored green, blue, magenta, and pink, respectively, and the peptides are shown as yellow tubes with side chains in stick representation. Antibody side chains engaging in hydrogen bonds (orange dashes) and key interacting aromatic residues are also shown as sticks. b The paratopes of Fab395 aligned to that of Fab311 based on CDR H2 (grey) are displayed as cartoons with their CDRs colored as shown, with the schematic of the Fab395 epitope structural motif also indicated below. The side chains of ${ }^{\mathrm{H}} \mathrm{Trp}^{52}, \mathrm{H}_{\mathrm{Tyr}}{ }^{98}$, and ${ }^{\mathrm{L}} \mathrm{Tyr}^{94},{ }^{\mathrm{H}} \mathrm{Cys}^{29}$, and ${ }^{\mathrm{H}} \mathrm{Cys}^{32}$ are highlighted as sticks (also with a surface representation for ${ }^{\mathrm{L}} \mathrm{Tyr}^{94}$ ). The peptides are shown as tubes with side chains as sticks and colored as indicated. c Side chains of residues involving in conserved molecular interactions from CDR H1 (green) and $\mathrm{H} 2$ (blue) and the peptides are shown as sticks. The peptide bound to Fab395 is colored magenta, whereas others are in yellow. Hydrogen bonds are displayed as orange dashes.

engineering of anti-NANP mAbs, including antibody evolution using yeast display technologies.

Likewise, structural analysis reveals features on both the paratopes and epitopes that may contribute to low-affinity antibody and, consequently, poor protection. One correlate that we observe is that the high-affinity protective mAbs all recognize epitopes with secondary structural motifs, consisting of a type I $\beta$-turn and Asn pseudo $3_{10}$ turn, which represent the repeating unit of the long-range spiral form of rsCSP (Fig. 6). Conversely, low affinity, less protective antibodies possess epitopes with few to no structural motifs (Table 2, and Fig. 6). A restricted binding groove, and consequently short epitope with a single type I 
a Fab224 binding groove

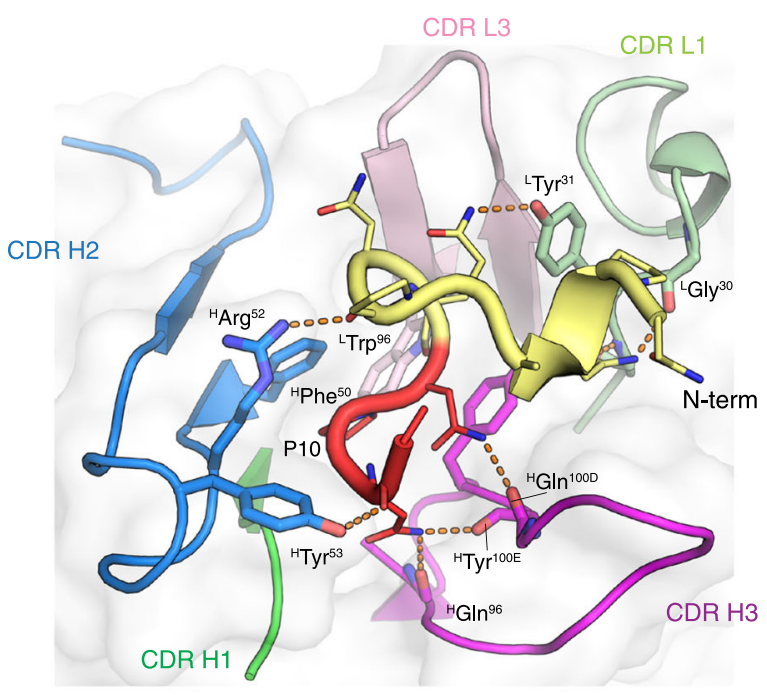

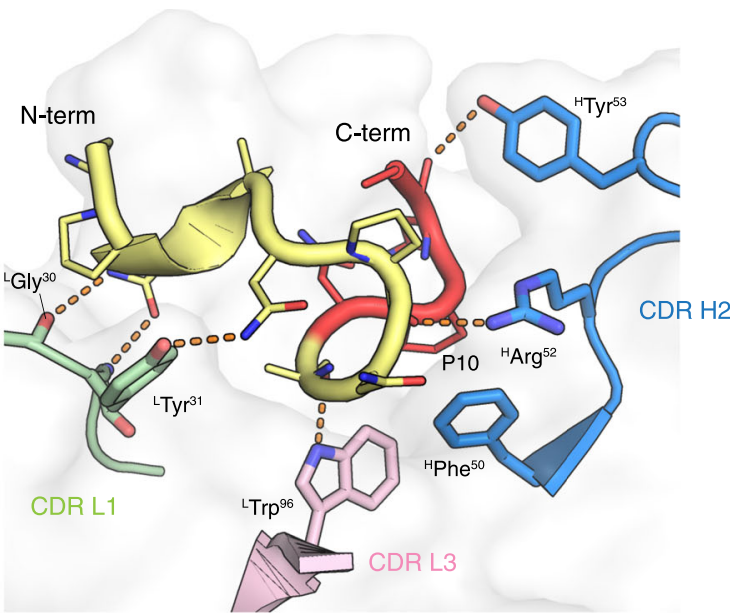

\begin{tabular}{ll|l|l|} 
Fab224 epitope $\quad \mathrm{N}-\mathrm{NPNA}$ & NPNA & NPNA & N C \\
\hline
\end{tabular} b Fab399 binding groove

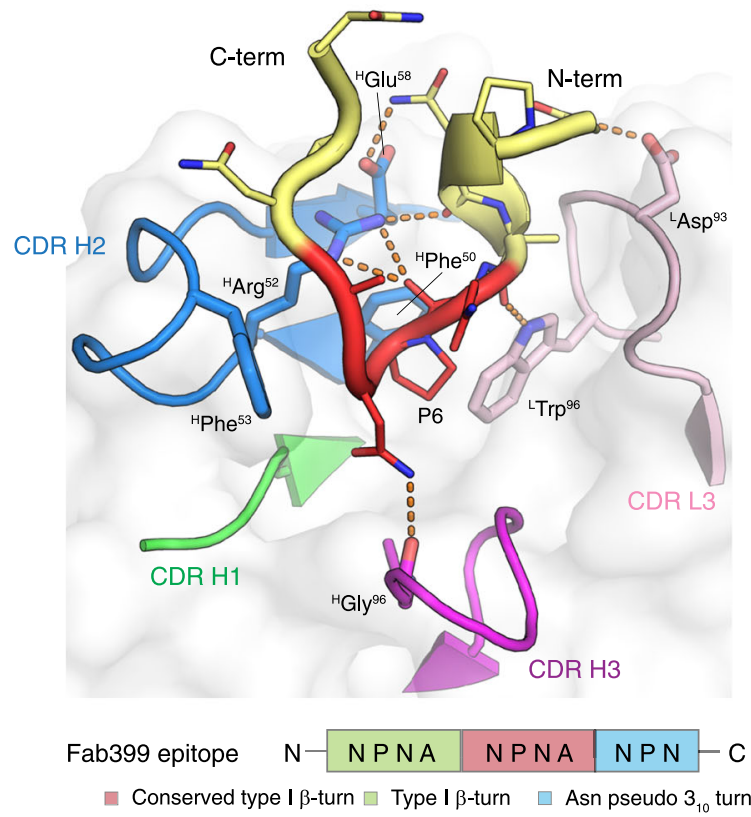

c Fab366 binding groove

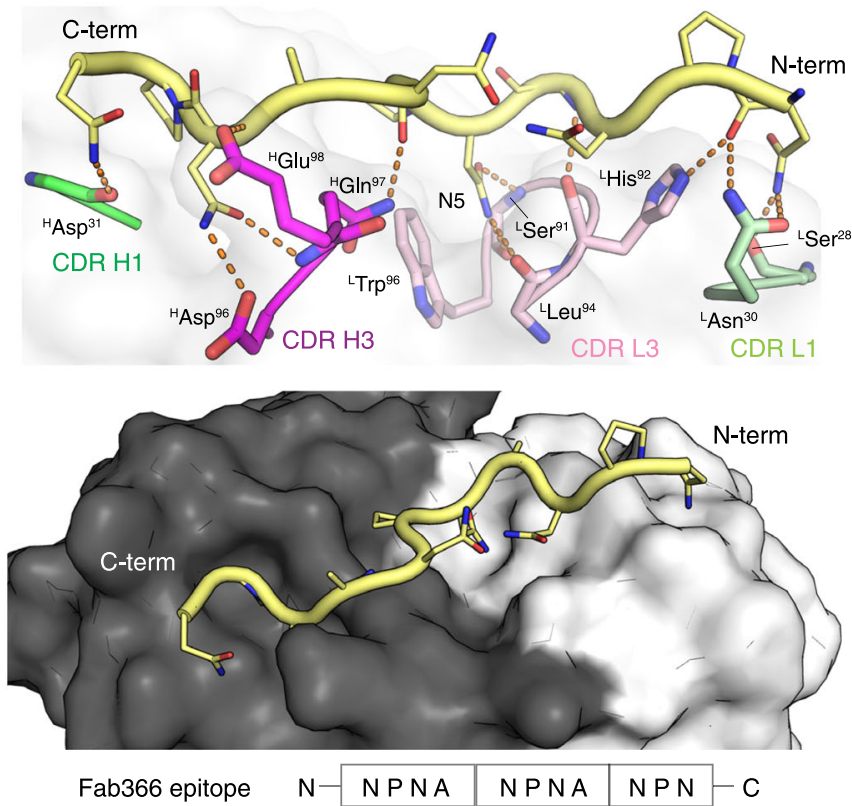

Fig. 5 The paratopes of Fab224, 399, and 366. a Crystal structure of Fab224 encoded by the IGHV3-49 gene and its epitope are shown as cartoons with residues where the main chain and side chain engage in hydrogen bonds (orange dashes) shown as sticks. The Fab cartoon representation is embedded in a transparent white surface rendering. Only the CDRs involved in the binding groove are displayed and colored green, blue, magenta, light green, and pink for $\mathrm{CDR} \mathrm{H1}, \mathrm{H} 2, \mathrm{H3}$, L1, and L3, respectively. A schematic of the epitope is also illustrated below. $\mathbf{b}$ The binding groove of Fab399 encoded by the IGHV3-49 gene. c The paratope of Fab366 encoded by the IGHV1-2 gene. All representations and coloring schemes for (b) and (c) are as in (a). In addition, a surface representation of Fab366 is shown, where the black and white surfaces represent the heavy and light chains, respectively.

$\beta$-turn, likely contributes to the low affinity of Fab395 (Figs. $4 \mathrm{~b}$ and 6). On the other hand, the low affinity and less protective Fab366 recognizes an extended conformation of NANP repeats that lack any secondary structural motifs with its shallow groove (Figs. 5c, 6). Intriguingly, the non-protective and low-affinity Fab1450 146 also shares similar features of utilizing a shallow groove to bind an extended NANP epitope. Consequently, structural motifs such as the repeating type I $\beta$-turn and Asn pseudo $3_{10}$ turn as in the spiral rsCSP could be incorporated into the design of next-generation immunogens, also with shorter length designs to prevent homotypic interactions. Future studies to explore anti-NANP mAbs from different germline genes and/ or immunization trials will help verify this hypothesis and/or contribute additional structural properties that influence binding affinity and in vivo protection. Other factors, such as pharmacokinetics, may impact antibody protective capacity in vivo and require further examination. Overall, the findings here should aid in defining the optimal characteristics of anti-NANP antibodies for therapeutic use, and also guide the design of more effective vaccines against malaria.

\section{Methods}

Antibody isolation, cloning, and sequencing. Antibody isolation, cloning, and sequencing were performed in a previous study using the protocol outlined in the 

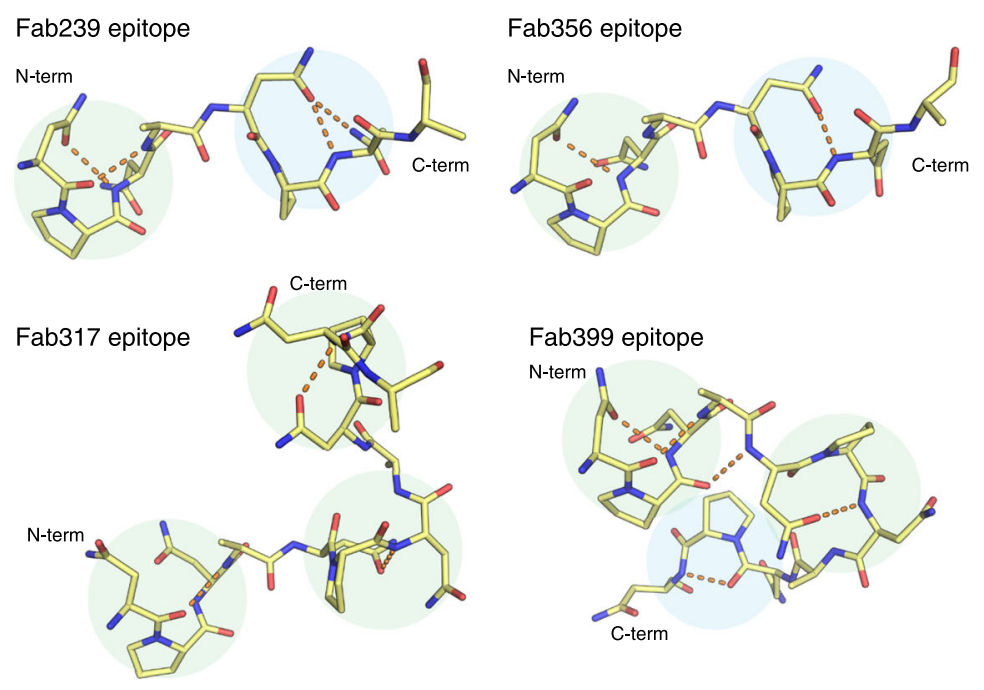

Fab364 epitope
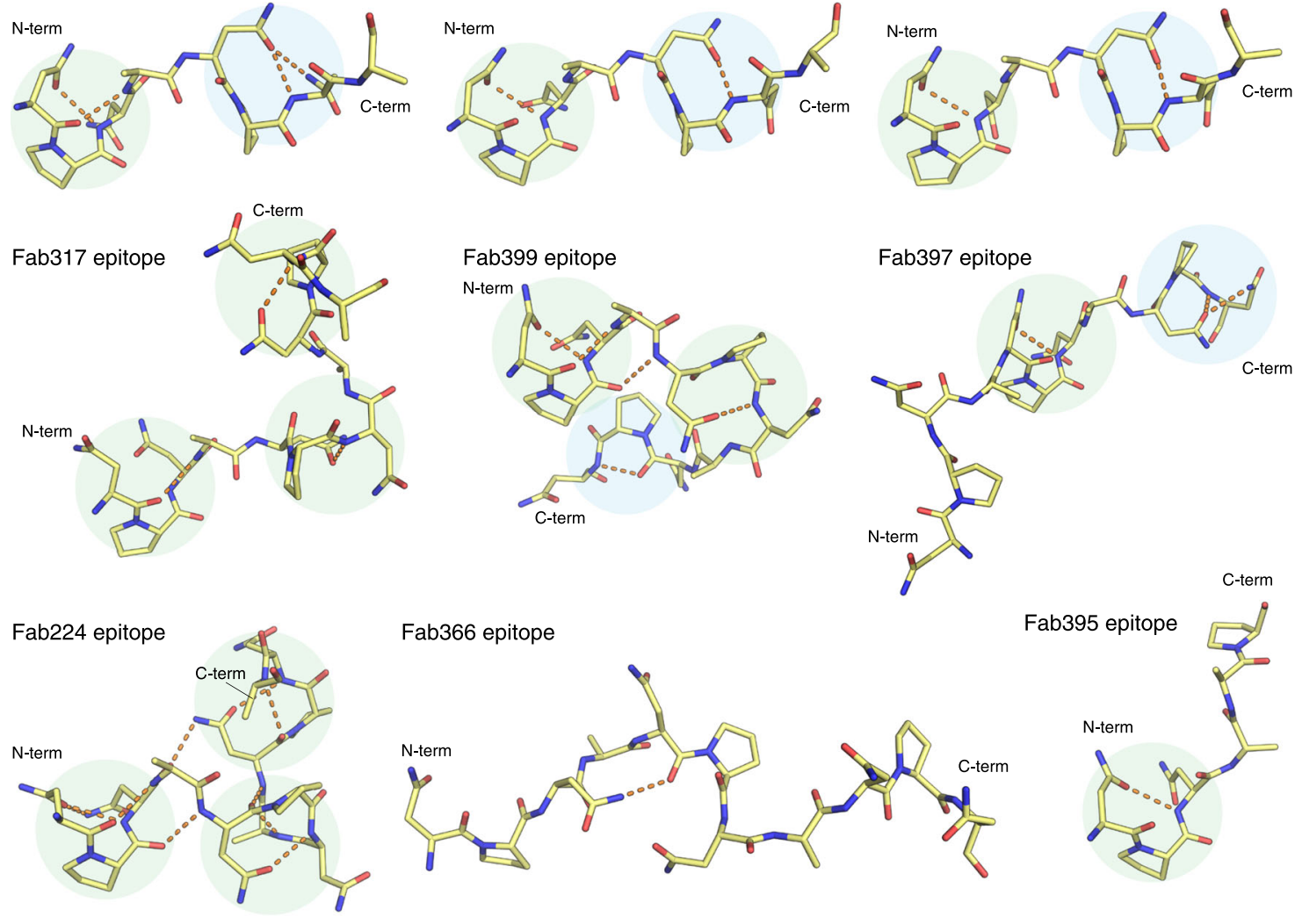

Fab366 epitope
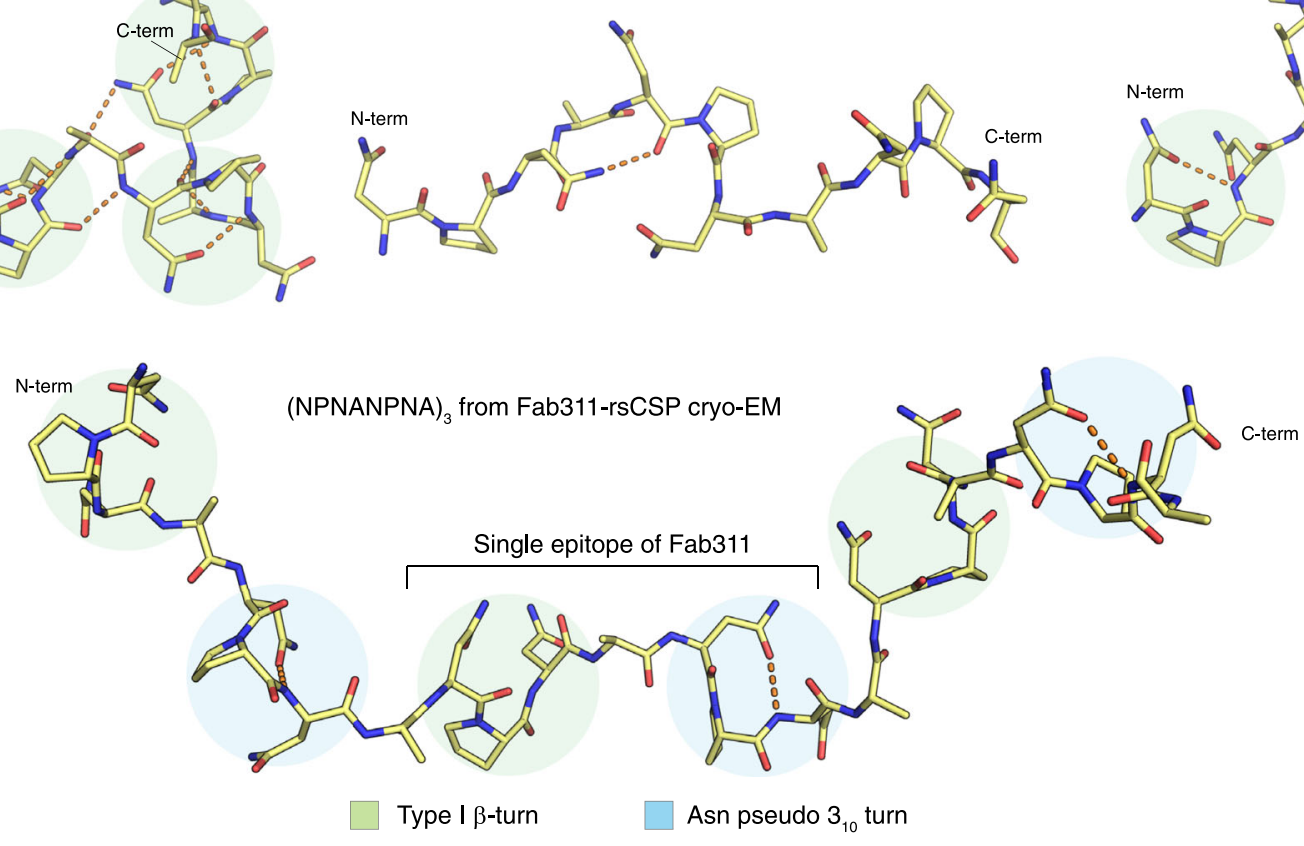

Fig. 6 Summary of the epitope conformations for all antibodies analyzed in this study. The peptide epitopes are represented with yellow sticks, and the hydrogen bonds are shown in orange dashes. The type I $\beta$-turns and Asn pseudo $3_{10}$ turns are highlighted with green and blue circles. All peptides were aligned based on the first type I $\beta$-turn that is present in the structure, except for the epitope of Fab366 which was aligned to the overall epitope of Fab239. The epitope of Fab317 and Fab311 were obtained from the crystal and cryo-EM structures from previous studies 27,28 (PDB ID: 6AXL and 6MB3, respectively).

original paper ${ }^{42}$. Briefly, peripheral blood mononuclear cells (PBMCs) were stained with the following mAbs: anti-CD3-FITC (BioLegend, cat\# 300406, clone UCHT1), anti-CD14-FITC (BioLegend, cat\# 325604, clone HCD14), anti-CD19-BV421 (BioLegend, cat\# 302234, clone HIB19), anti-CD20-PerCP/cy5.5 (BD, cat\# 340955 , clone L27), anti-CD27-BV510 (BioLegend, cat\# 302836, clone O323), anti-CD38$\mathrm{PE} / \mathrm{cy} 7$ (BioLegend, cat\# 356607, clone HB-7), anti-IgA-FITC (Miltenyi, cat\# 130113-475, clone IS11-8E10), anti-IgM-APC/cy7 (BioLegend, cat\# 314520, clone MHM-88). $\mathrm{IgG}^{+}$plasmablasts were single-cell sorted into 96-well PCR plates containing hypotonic buffer ( $330 \mathrm{nM}$ dNTPs (NEB), $1 \mu \mathrm{g} / \mathrm{ml} \mathrm{BSA} \mathrm{(NEB),} 2 \mathrm{mM}$ DTT (Sigma), 0.5\% IGEPAL-630 and 500 unit $/ \mathrm{mL}$ of Ribolock (Thermo)) based on gating for $\mathrm{CD} 19^{+} \mathrm{CD} 20^{-} \mathrm{CD} 27^{+} \mathrm{CD} 38^{++} \mathrm{IgA}^{-} \mathrm{IgM}^{-}$cells. Generation of barcoded cDNA, PCR amplification, and 454 sequencing of IgG were performed as described $^{42}$ with the following modifications: Biotinylated Oligo(dT) and RT maxima H- (Fisher Scientific Company) were used for reverse transcription, cDNA was extracted using Streptavidin C1 beads (Life Technologies), DNA concentrations were determined using qPCR (KAPA SYBR ${ }^{\circledast}$ FAST qPCR Kit for Titanium, Kapabiosystems), and amplicons were sequenced using Roche 454 Titanium sequencing. Barcode assignment, sequence assembly, assignment of $\mathrm{V}(\mathrm{D}) \mathrm{J}$, and identification of mutations were performed as described ${ }^{42}$ except for the following modifications: a minimum coverage of 10 reads was required for each heavy and light chain assembly to be acceptable. Heavy (light) reads were required to assemble unique contigs within a well. In cases where there was more than one contig, we rejected the well from consideration unless one of the contigs included at least $90 \%$ of the reads. V(D)J assignment and mutation identification was performed using an implementation of SoDA ${ }^{43}$. The sequences of $\mathrm{mAb} 239, \mathrm{mAb} 337$,

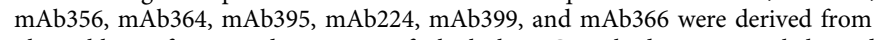
plasmablasts of protected vaccinees, of which the IgG antibody sequences belonged to expanded plasmablast IgG sequence families within the respective vaccinee's repertoire. The collection of plasmablasts was part of the phase 2a clinical trial of RTS,S/AS01 vaccine with fractional third and fourth dose $e^{31}$, of which the protocol was approved by the Walter Reed Army Institute of Research (WRAIR) Institutional Review Board and the Western Institutional Review Board, and written informed consent was obtained from each subject before study procedures were initiated (Clinical Trials.gov identifier: NCT01857869). None of this work was performed as part of this study, but the antibody sequences used here were derived from that study. 
Trp-Pro interaction ( $\mathrm{CH}-\pi /$ van der Waals)

Fab239, 311, 356,

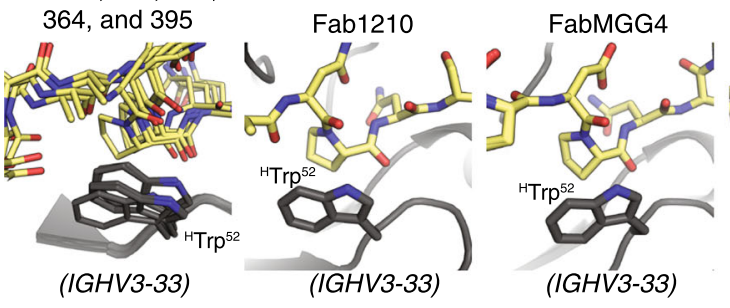

Trp-Asn interaction ( $\pi-\pi$ interaction)
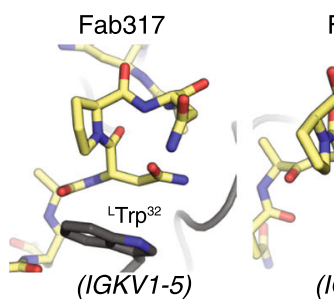

Fab397
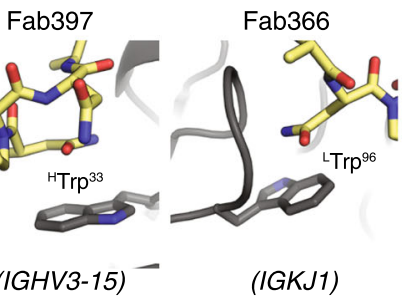

(IGHV3-15)

$(\mid G K J 1)$
Trp: $\mathrm{H}$-bond to peptide backbone
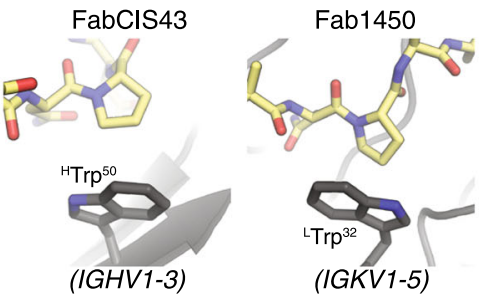

Phe-Pro interaction $(\mathrm{CH}-\pi /$ van der Waals)
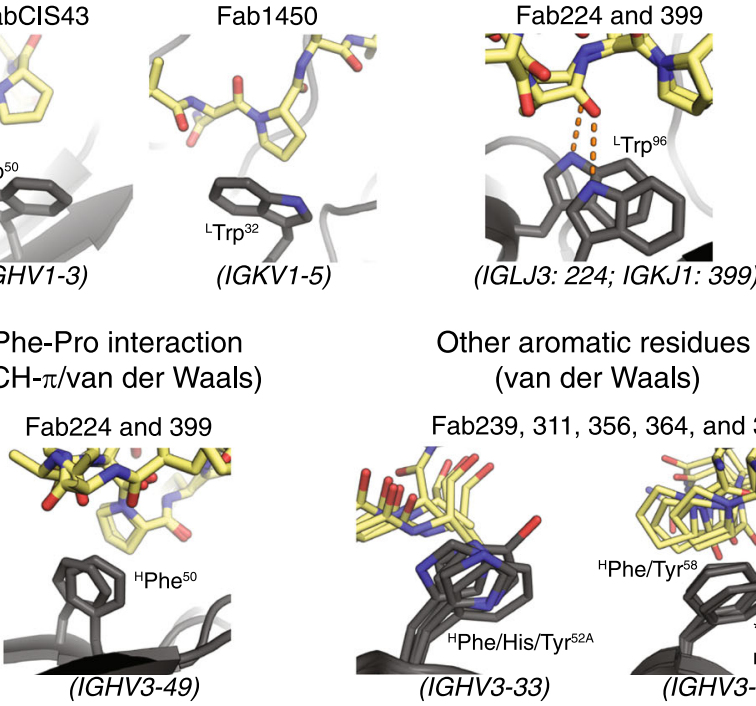

\section{Other aromatic residues \\ (van der Waals)}

Fig. 7 Summary of interactions between Fab aromatic residues and the NANP or junction region. The Fab aromatic residues and CSP peptides are shown in black and yellow sticks, respectively. Hydrogen bonds are represented as orange dashes. Residue identity and number (with $\mathrm{H}$ and $\mathrm{L}$ for heavy and light chain) and the corresponding antibody germline gene is indicated. Crystal structures of Fab1210, MGG4, CIS43, 1450, and 317 and 397 were obtained from previous studies $22,23,26,28,29$ (PDB ID: 6D01, 6BQB, 6B50, 6D11, 6AXL, and 6UC5, respectively).

\begin{abstract}
Antibody production. For protection studies, all $\mathrm{mAbs}$ were made as IgG1 and expressed in Chinese hamster ovary cells (ExpiCHO; Thermo Fisher Scientific). The mAbs were purified using HiTrap Protein A HP column (GE Healthcare), followed by size exclusion chromatography (Superdex 200 16/90; GE Healthcare) and washed with $0.5 \mathrm{M}$ Arginine in Dulbecco's PBS pH 7.4 (DPBS: Thermo Fisher), which has been shown to be effective in removing endotoxins from IgGs ${ }^{44}$. The absence of endotoxin contamination was determined using Endosafe ${ }^{\circledast}$ nexgenPTS $^{\mathrm{m}}$ portable endotoxin testing system (Charles River). For structural and biophysical characterizations, all Fabs were expressed in ExpiCHO cells and purified using a HiTrap Protein G HP column (GE Healthcare) followed by size exclusion chromatography as used for the IgG1 but in Tris Buffered Saline (TBS: $50 \mathrm{mM}$ Tris $\mathrm{pH} 8.0,137 \mathrm{mM} \mathrm{NaCl}, 3.6 \mathrm{mM} \mathrm{KCl}$ ). rsCSP was expressed in E. coli (SHUFFLE cells; New England Biolabs) and purified as previously reported ${ }^{45}$. In short, a pET28a plasmid containing the rsCSP construct was used to transform E. coli SHUFFLE competent cells. A single colony was used to start a $50-\mathrm{mL}$ overnight culture. Two 1-L cultures were inoculated the next day with $25 \mathrm{~mL}$ overnight culture and were grown at $37^{\circ} \mathrm{C}$. When the optical density at $600 \mathrm{~nm}$ reached a value of 1 , the cultures were induced with $1 \mathrm{mM}$ isopropyl $\beta$-D-1-

thiogalactopyranoside for $6 \mathrm{~h}$. The cells then were harvested and lysed by microfluidization. The lysate was incubated overnight with $\mathrm{Ni}$ cOmplete ${ }^{\mathrm{TM}}$ resin (Roche) and was eluted using a buffer containing $200 \mathrm{mM}$ imidazole. Typically, $10 \mathrm{mg}$ of protein was obtained per $2 \mathrm{~L}$ of culture. All synthetic NANP peptides in this study were purchased from Innopep Inc. (San Diego, CA) with the purity level of $95 \%$ for structural studies and $98 \%$ for biophysical measurements.
\end{abstract}

Assessment of in vivo protection. Studies using mice were carried out using 6-8 weeks old C57BL/6 females, maintained at the animal facility of the Johns Hopkins Bloomberg School of Public Health. Mice rooms are kept at $40-60 \%$ relative humidity at a temperature of $68-79^{\circ} \mathrm{F}$ with at least 10 room air changes per hour. Mice have a cycle of $14 \mathrm{~h}$ light and $10 \mathrm{~h}$ darkness. The assays using mice were performed in strict accordance with the recommendations in the Guide for the Care and Use of Laboratory Animals of the National Institutes of Health. The protocol was approved by the Animal Care and Use Committee of the Johns Hopkins University, protocol number MO18H419. Experiments were performed with the protocol established previously $y^{32,33}$. Briefly, to measure liver burden, mice $(N=5)$ were IV injected with $100 \mu \mathrm{g}$ of $\mathrm{mAb}$ per mouse and, $16 \mathrm{~h}$ later, challenged IV with 2000 P. berghei transgenic sporozoites expressing the P. falciparum CSP and luciferase. $42 \mathrm{~h}$ after challenge, mice were injected IP with $100 \mu \mathrm{l}$ of D-luciferin $(30 \mathrm{mg} / \mathrm{mL})$, having previously been anesthetized by exposure to isoflurane. Bioluminescence in the liver was measured using an IVIS Spectrum (Perkin Elmer). For the blood-stage parasitemia study, mice $(N=6)$ were passively immunized with 100 or $300 \mu \mathrm{g}$ of $\mathrm{mAb}$ and, $16 \mathrm{~h}$ later, recipient mice and controls were anesthetized with $2 \%$ Avertin prior to challenge by a 10 -minute exposure to the bites of 7 mosquitoes of which 5 on average are infected with the transgenic parasite. Parasite infection of red blood cells was assessed from day 4 after a challenge by microscopic observation of blood smears.
Isothermal titration calorimetry. ITC experiments were performed on a MicroCal Auto-iTC200 (GE Healthcare). Prior to the measurement, all Fabs were extensively dialyzed against DPBS. The peptides were placed in the syringe at a concentration of $\sim 150 \mu \mathrm{M}$ for Ac-NPNA NPNA-NH ${ }_{2}\left(\mathrm{NPNA}_{2} ; 300 \mu \mathrm{M}\right.$ for Fab224 and $\sim 500 \mu \mathrm{M}$ for Fab399), $\sim 60 \mu \mathrm{M}$ (IGHV3-33 Fabs) or $\sim 140 \mu \mathrm{M}$ (other Fabs) for AcNPNANPNA NPNANPNA-NH ${ }_{2}\left(\mathrm{NPNA}_{4}\right), \sim 40 \mu \mathrm{M}$ (IGHV3-33 Fabs) or $\sim 60 \mu \mathrm{M}$ (other Fabs) for Ac-NPNANPNA NPNANPNA NPNANPNA-NH ${ }_{2}\left(\mathrm{NPNA}_{6}\right)$, whereas the concentration of Fab in the cell was $\sim 10 \mu \mathrm{M}$ for all experiments. The Fab and peptide concentrations were determined by UV absorbance at $280 \mathrm{~nm}$ and $205 \mathrm{~nm}$, where the molar extinction coefficients for the peptides at $205 \mathrm{~nm}$ were estimated using the Protein Parameter Calculator [http://nickanthis.com/tools/ a205.html ${ }^{46}$. The titrations were all performed with peptides in the syringe and antibodies in the cell and consisted of 16 injections of $2.45 \mu \mathrm{l}$ peptide for experiments with $\mathrm{NPNA}_{2}$ and 32 injections for other experiments at a rate of $0.5 \mu \mathrm{l} / \mathrm{s}$ at $120 \mathrm{~s}$ time intervals, with injection duration of $4.9 \mathrm{~s}$, injection interval of $180 \mathrm{~s}$, and reference power of $5 \mu \mathrm{Cal}$. Experiments were conducted in triplicate $(N=3)$ at $25^{\circ} \mathrm{C}$. Fitting of the integrated titration peaks was performed with Origin 7.0 software using a single-site binding model. The first data point was excluded from the fit as common practice.

Bio-layer interferometry. The binding of all Fabs to biotinylated-rsCSP was measured using bio-layer interferometry (Octet Red; Pall ForteBio). BiotinylatedrsCSP were loaded onto streptavidin biosensors (Pall ForteBio, cat No 18-5019) at $10 \mu \mathrm{g} / \mathrm{mL}$ in kinetics buffer (TBS $+0.002 \%$ Tween 20 and $0.01 \%$ BSA). The loaded sensors were dipped into solutions containing dilutions of each Fab in Kinetics buffer at a concentration of $1000,500,250,125$, and $62.5 \mathrm{nM}$, respectively (except for Fab317, the serial dilution concentrations are 250, 125, 62.5, 31.25, 15.63 nM, respectively). The binding experiments were performed with the following steps: 1) baseline in kinetics buffer for $60 \mathrm{~s} ; 2$ ) loading of rsCSP for $60 \mathrm{~s} ; 3$ ) baseline for $60 \mathrm{~s} ; 4$ ) association of antibody for $60 \mathrm{~s}$; and 5) dissociation of antibody into kinetics buffer for $120 \mathrm{~s}$. A reference well with no rsCSP loaded onto the sensor was run in all experiments and subtracted from sample wells to correct for drift and buffer evaporation. Octet assays were carried out at $25^{\circ} \mathrm{C}$. Data were analyzed using the Octet Red Data Analysis software version 9.0 and fitted with a 1:1 binding model.

Differential scanning calorimetry. The thermal stability of all IgG1 in Dulbecco's PBS (Thermo Fisher) from 20 to $110^{\circ} \mathrm{C}$ was measured using a MicroCal VPCapillary calorimeter (Malvern) at a scanning rate of $90^{\circ} \mathrm{C} / \mathrm{h}$. Data were analyzed using the VP-Capillary DSC automated data analysis software and fitted to a nontwo-state model.

X-ray crystallography and structural analysis. Fabs $239,356,364,395,224,250$, 399 , and 366 were concentrated to $10 \mathrm{mg} / \mathrm{ml}$ and mixed with either $\mathrm{NPNA}_{2}$, $\mathrm{NPNA}_{3}, \mathrm{NPNA}_{4}$, or $\mathrm{NPNA}_{6}$ peptide in a 1:5 molar ratio of Fab to peptide. Six substitutions and one deletion (from ${ }^{112}$ SSASTKG $^{118}$ to ${ }^{112}$ VSRRLP $^{117}$ ) were introduced into the elbow region of Fab395 and Fab366 heavy chains, and different 
mutations (from ${ }^{112}$ SSASTKG $^{118}$ to ${ }^{112} \mathrm{FNQIKG}^{117}$ ) were introduced to the elbow region of Fab364 heavy chain to stabilize the Fab and facilitate crystallization as shown in previous work ${ }^{47}$. Additionally, Fab239-NPNA 2 , Fab364-NPNA 2 and Fab250-NPNA 3 co-complexes were mixed with Streptococcal immunoglobulin Gbinding protein $\mathrm{G}$ (domain III) in the Fab to protein $\mathrm{G}$ ratio of 1:1. Domain III of protein $\mathrm{G}$ has also previously been shown to enhance the crystallizability of Fabs ${ }^{48}$. Crystal screening of Fab-peptide complexes was performed using our highthroughput, robotic CrystalMation system (Rigaku) at The Scripps Research Institute, using the sitting drop vapor diffusion method with a $35 \mu \mathrm{L}$ reservoir solution and each drop consisting of $0.1 \mu \mathrm{L}$ protein $+0.1 \mu \mathrm{L}$ precipitant. Fab239$\mathrm{NPNA}_{2}$ co-crystals were grown in $0.2 \mathrm{M} \mathrm{NaCl}$, and $20 \%$ (w/v) PEG 3350 at $20^{\circ} \mathrm{C}$ as precipitant and were cryoprotected in $20 \%$ ethylene glycol. Fab356-NPNA 2 crystals and Fab366-NPNA 3 grew in 40\% PEG-600, and $0.1 \mathrm{M}$ CHES pH 9.5 with final $\mathrm{pH}$ of 9.6 at $4^{\circ} \mathrm{C}$. Fab364-NPNA 2 crystals grew in 20\% PEG-8000, and $0.05 \mathrm{M} \mathrm{KH}_{2} \mathrm{PO}_{4}$ at $4{ }^{\circ} \mathrm{C}$ and were cryoprotected in $20 \%$ glycerol. Fab395-NPNA ${ }_{2}$ crystals grew in $30 \%$ PEG-4000, $0.2 \mathrm{M}$ ammonium acetate, and $0.1 \mathrm{M}$ sodium citrate $\mathrm{pH} 5.6$ at $20^{\circ} \mathrm{C}$. Fab224-NPNA 4 crystals and Fab239-NPNA 4 grew in 20\% PEG-6000, and $0.1 \mathrm{M}$ HEPES pH 7.0 at $20^{\circ} \mathrm{C}$ and were cryoprotected in $20 \%$ ethylene glycol. Fab399-NPNA 3 crystals grew in 20\% PEG 3350, and $0.2 \mathrm{M}$ potassium fluoride $\mathrm{pH}$ 7.2 at $20^{\circ} \mathrm{C}$ and were cryoprotected in $20 \%$ ethylene glycol. Fab250-NPNA 4 crystals grew in $1.6 \mathrm{M}$ ammonium sulfate, and $0.1 \mathrm{M}$ citric acid $\mathrm{pH} 4.0$ at $20^{\circ} \mathrm{C}$ and were cryoprotected in $20 \%$ glycerol. Fab399-NPNA 6 crystals grew in 50\% MPD, $0.2 \mathrm{M}$ ammonium dihydrogen phosphate, $0.1 \mathrm{M}$ Tris $\mathrm{pH} 8.5$ at $20^{\circ} \mathrm{C}$. X-ray diffraction data were collected at the Advanced Proton Source beamline 23ID-B or beamline 23IDD, or at the Stanford Synchrotron Radiation Lightsource beamline 12-2, and processed and scaled using the HKL-2000 package ${ }^{49}$. The structures were determined by molecular replacement using $\mathrm{Phaser}^{50}$. Structure refinement was performed using phenix.refine ${ }^{51}$ and iterations of refinement using Coot $^{52}$. Aminoacid residues of the Fabs were numbered using the Kabat system, and the structures were validated using MolProbity ${ }^{53}$. For structural analysis, buried surface areas (BSAs) were calculated with the program $\mathrm{MS}^{54}$, and hydrogen bonds were assessed with the program HBPLUS 55 .

Statistical analysis. The parasite liver burden load data ( $N=5$ mice) were compared for significance using a two-sided Mann-Whitney $\mathrm{U}$ test, whereas the blood-stage parasitemia data $(N=6$ mice $)$ were analyzed using the log-rank test where $p<0.05\left(^{*}\right)$ and $p<0.01(* *)$ indicated levels of statistically significant differences. The liver burden data were reported as the geometric mean of the total flux \pm SD (Fig. 1a). All statistical parameters for the mouse in vivo studies were calculated with the Hmisc (liver burden data), and the survival and survminer packages (parasitemia data), and the graphs were plotted with the ggplot2 package in R. Bootstrapping for the linear regression models was performed with the caret package and also plotted with the ggplot2 in R. Each ITC experiment was performed with three replicates $(N=3)$, and the data are reported as the arithmetic mean $\pm \mathrm{SD}$.

Reporting summary. Further information on research design is available in the Nature Research Reporting Summary linked to this article.

\section{Data availability}

The crystal structures of all Fab-peptide complexes have been deposited in the Protein Data Bank with accession codes:

6W00 (Fab239-NPNA ${ }_{2}$,

6W05 (Fab356-NPNA ${ }_{2}$,

6WFW (Fab364-NPNA $)_{2}$

6WFX (Fab395-NPNA $\left.{ }_{2}\right)$

6WFY (Fab224-NPNA ${ }_{4}$,

6WFZ (Fab399-NPNA ${ }_{3}$,

6WG0 (Fab366-NPNA $)_{3}$,

6WG1 (Fab399-NPNA A $_{6}$,

6WG2 (Fab239-NPNA 4 )

Crystal structures of Fab1210, MGG4, CIS43, 1450, and 317 and 397 used in Figs. 6-7 were obtained from previous studies 22,23,26,28,29 (PDB ID: 6D01, 6BQB, 6B5O, 6D11, $6 \mathrm{AXL}$, and 6UC5, respectively). Cryo-EM structures of Fab311 in complex with rsCSP used in Fig. 6 were also obtained from our previous study ${ }^{27}$ (PDB ID: 6MB3). Source data are provided with this paper.

Received: 20 July 2020; Accepted: 13 January 2021;

Published online: 16 February 2021

\section{References}

1. Toé, K. H. et al. Increased pyrethroid resistance in malaria vectors and decreased bed net effectiveness, Burkina Faso. Emerg. Infect. Dis. 20, 1691 (2014).
2. Hamilton, W. L. et al. Evolution and expansion of multidrug-resistant malaria in southeast Asia: a genomic epidemiology study. Lancet Infect. Dis. 19, 943-951 (2019).

3. Adepoju, P. RTS, S malaria vaccine pilots in three African countries. Lancet 393, 1685 (2019).

4. Ancsin, J. B. \& Kisilevsky, R. A binding site for highly sulfated heparan sulfate is identified in the $\mathrm{N}$ terminus of the circumsporozoite protein: significance for malarial sporozoite attachment to hepatocytes. J. Biol. Chem. 279, 21824-21832 (2004).

5. Coppi, A. et al. The malaria circumsporozoite protein has two functional domains, each with distinct roles as sporozoites journey from mosquito to mammalian host. J. Exp. Med. 208, 341-356 (2011).

6. Coppi, A. et al. Heparan sulfate proteoglycans provide a signal to Plasmodium sporozoites to stop migrating and productively invade host cells. Cell Host Microbe 2, 316-327 (2007).

7. Ménard, R. et al. Circumsporozoite protein is required for development of malaria sporozoites in mosquitoes. Nature 385, 336 (1997).

8. Zavala, F. et al. Rationale for development of a synthetic vaccine against Plasmodium falciparum malaria. Science 228, 1436-1440 (1985).

9. Schwenk, R. et al. Immunization with the RTS, S/AS malaria vaccine induces IFN- $\gamma+\mathrm{CD} 4 \mathrm{~T}$ cells that recognize only discrete regions of the circumsporozoite protein and these specificities are maintained following booster immunizations and challenge. Vaccine 29, 8847-8854 (2011).

10. Bahl, A. et al. PlasmoDB: the Plasmodium genome resource. A database integrating experimental and computational data. Nucleic Acids Res. 31, 212-215 (2003).

11. Bowman, N. M. et al. Comparative population structure of Plasmodium falciparum circumsporozoite protein NANP repeat lengths in Lilongwe, Malawi. Sci. Rep. 3, 1990 (2013).

12. Dame, J. B. et al. Structure of the gene encoding the immunodominant surface antigen on the sporozoite of the human malaria parasite Plasmodium falciparum. Science 225, 593-599 (1984).

13. Enea, V. et al. DNA cloning of Plasmodium falciparum circumsporozoite gene: amino acid sequence of repetitive epitope. Science 225, 628-630 (1984).

14. Casares, S., Brumeanu, T.-D. \& Richie, T. L. The RTS, S malaria vaccine. Vaccine 28, 4880-4894 (2010).

15. Olotu, A. et al. Four-year efficacy of RTS, S/AS01E and its interaction with malaria exposure. N. Engl. J. Med 368, 1111-1120 (2013).

16. Olotu, A. et al. Seven-year efficacy of RTS, S/AS01 malaria vaccine among young African children. N. Engl. J. Med 374, 2519-2529 (2016).

17. RTS, S. Clinical Trial Partnerships. Efficacy and safety of RTS, S/AS01 malaria vaccine with or without a booster dose in infants and children in Africa: final results of a phase 3 , individually randomised, controlled trial. Lancet $\mathbf{3 8 6}$, $31-45$ (2015)

18. White, M. T. et al. Immunogenicity of the RTS,S/AS01 malaria vaccine and implications for duration of vaccine efficacy: secondary analysis of data from a phase 3 randomised controlled trial. Lancet Infect. Dis. 15, 1450-1458 (2015).

19. Venkatraman, N. et al. Phase I assessments of first-in-human administration of a novel malaria anti-sporozoite vaccine candidate, $\mathrm{R} 21$ in matrix-M adjuvant, in UK and Burkinabe volunteers. Preprint at https://doi.org/ $10.1101 / 19009282(2019)$.

20. Hoffman, S. L. et al. Development of a metabolically active, non-replicating sporozoite vaccine to prevent Plasmodium falciparum malaria. Hum. Vaccin 6 , 97-106 (2010).

21. Ishizuka, A. S. et al. Protection against malaria at 1 year and immune correlates following PfSPZ vaccination. Nat. Med 22, 614-623 (2016).

22. Kisalu, N. K. et al. A human monoclonal antibody prevents malaria infection by targeting a new site of vulnerability on the parasite. Nat. Med. 24, 408 (2018).

23. Tan, J. et al. A public antibody lineage that potently inhibits malaria infection through dual binding to the circumsporozoite protein. Nat. Med. 24, 401 (2018)

24. Murugan, R. et al. Evolution of protective human antibodies against Plasmodium falciparum circumsporozoite protein repeat motifs. Nat. Med 26, 1135-1145 (2020)

25. Oyen, D. et al. Structure and mechanism of monoclonal antibody binding to the junctional epitope of Plasmodium falciparum circumsporozoite protein. PLoS Pathog. 16, e1008373 (2020).

26. Imkeller, K. et al. Antihomotypic affinity maturation improves human B cell responses against a repetitive epitope. Science 360, 1358-1362 (2018).

27. Oyen, D. et al. Cryo-EM structure of $P$. falciparum circumsporozoite protein with a vaccine-elicited antibody is stabilized by somatically mutated inter-Fab contacts. Sci. Adv. 4, eaau8529 (2018).

28. Oyen, D. et al. Structural basis for antibody recognition of the NANP repeats in Plasmodium falciparum circumsporozoite protein. Proc. Natl Acad. Sci. USA 114, E10438-E10445 (2017).

29. Pholcharee, T. et al. Diverse antibody responses to conserved structural motifs in Plasmodium falciparum circumsporozoite protein. J. Mol. Biol. 432, 1048-1063 (2020). 
30. Triller, G. et al. Natural parasite exposure induces protective human antimalarial antibodies. Immunity 47, 1197-1209. e1110 (2017).

31. Regules, J. A. et al. Fractional third and fourth dose of RTS,S/AS01 malaria candidate vaccine: a phase 2a controlled human malaria parasite infection and immunogenicity study. J. Infect. Dis. 214, 762-771 (2016).

32. Flores-Garcia, Y. et al. Optimization of an in vivo model to study immunity to Plasmodium falciparum pre-erythrocytic stages. Malar. J. 18, 426 (2019).

33. Raghunandan, R. et al. Characterization of two in vivo challenge models to measure functional activity of monoclonal antibodies to Plasmodium falciparum circumsporozoite protein. Malar. J. 19, 113 (2020).

34. Rouge, L. et al. Structure of CD20 in complex with the therapeutic monoclonal antibody rituximab. Science 367, 1224-1230 (2020).

35. Schofield, L. The circumsporozoite protein of Plasmodium: a mechanism of immune evasion by the malaria parasite? Bull. World Health Organ 68, 66-73 (1990).

36. Murugan, R. et al. Clonal selection drives protective memory B cell responses in controlled human malaria infection. Sci. Immunol. 3, eaap8029 (2018).

37. Cockburn, I. A. \& Seder, R. A. Malaria prevention: from immunological concepts to effective vaccines and protective antibodies. Nat. Immunol. 19, 1199-1211 (2018).

38. Kucharska, I. et al. Structural ordering of the Plasmodium berghei circumsporozoite protein repeats by inhibitory antibody 3D11. eLife $\mathbf{9}$, e59018 (2020).

39. Herrington, D. A. et al. Safety and immunogenicity in man of a synthetic peptide malaria vaccine against Plasmodium falciparum sporozoites. Nature 328, 257-259 (1987).

40. Nussenzweig, R. S. \& Nussenzweig, V. Antisporozoite vaccine for malaria: experimental basis and current status. Rev. Infect. Dis. 11, S579-S585 (1989).

41. Lewis, F. J. et al. Avid binding by B cells to the Plasmodium circumsporozoite protein repeat suppresses responses to protective subdominant epitopes. Preprint at https://doi.org/10.1101/2020.01.12.903682 (2020).

42. Tan, Y. C. et al. Barcode-enabled sequencing of plasmablast antibody repertoires in rheumatoid arthritis. Arthritis Rheumatol. 66, 2706-2715 (2014).

43. Volpe, J. M., Cowell, L. G. \& Kepler, T. B. SoDA: implementation of a 3D alignment algorithm for inference of antigen receptor recombinations. Bioinformatics 22, 438-444 (2006).

44. Ritzén, U., Rotticci-Mulder, J., Strömberg, P. \& Schmidt, S. R. Endotoxin reduction in monoclonal antibody preparations using arginine. J. Chromatogr. B: Anal. Technol. Biomed. Life Sci. 856, 343-347 (2007).

45. Schwenk, R. et al. IgG2 antibodies against a clinical grade Plasmodium falciparum CSP vaccine antigen associate with protection against transgenic sporozoite challenge in mice. PLoS ONE 9, el11020-e111020 (2014).

46. Anthis, N. J. \& Clore, G. M. Sequence-specific determination of protein and peptide concentrations by absorbance at $205 \mathrm{~nm}$. Protein Sci. 22, 851-858 (2013).

47. Bailey, L. J. et al. Locking the elbow: Improved antibody Fab fragments as chaperones for structure determination. J. Mol. Biol. 430, 337-347 (2018).

48. Derrick, J. P., Feavers, I. \& Maiden, M. C. Use of streptococcal protein G in obtaining crystals of an antibody Fab fragment in complex with a meningococcal antigen. Acta Crystallogr. D: Biol. Crystallogr. 55, 314-316 (1999).

49. Otwinowski, Z. \& Minor, W. Processing of X-ray diffraction data collected in oscillation mode. Methods Enzymol. 276, 307-326 (1997).

50. McCoy, A. J. et al. Phaser crystallographic software. J. Appl. Crystallogr. 40 , 658-674 (2007).

51. Adams, P. D. et al. PHENIX: a comprehensive Python-based system for macromolecular structure solution. Acta Crystallogr. D: Biol. Crystallogr. 66, 213-221 (2010).

52. Emsley, P., Lohkamp, B., Scott, W. G. \& Cowtan, K. Features and development of Coot. Acta Crystallogr D: Biol. Crystallogr 66, 486-501 (2010).

53. Chen, V. B. et al. MolProbity: all-atom structure validation for macromolecular crystallography. Acta Crystallogr D: Biol. Crystallogr. 66, $12-21$ (2010).
54. Connolly, M. L. The molecular surface package. J. Mol. Graph 11, 139-141 (1993).

55. McDonald, I. K. \& Thornton, J. M. Satisfying hydrogen bonding potential in proteins. J. Mol. Biol. 238, 777-793 (1994).

\section{Acknowledgements}

The authors thank Robyn Stanfield, Xueyong Zhu, and Xiaoping Dai (The Scripps Research Institute) for advice regarding crystal structure determination, and Randall MacGill and Ashley Birkett for careful reading and comments on the manuscript. This work was supported by PATH's Malaria Vaccine Initiative and the Bill \& Melinda Gates Foundation (grants no. OPP1170236 and INV-004923) under collaborative agreements with The Scripps Research Institute. Y.F-G. and F.Z. thank Bloomberg Philanthropies for continued support. We thank other members of the collaborative team who identified antibodies for further analysis from the Mal071 trial: Christian Ockenhouse, Ulrike Wille-Reece and Scott Gregory (PATH), Erik Jongert and Robert van den Berg (GSK), Bill Robinson, Xiaomu Chen, Kevin Williamson, Dongkyoon Kim, and Yvonne Leung (Atreca), and Sheetij Dutta, Jason Regules, Elke Bergmann-Leitner and Michele Spring (WRAIR).

\section{Author contributions}

Conceptualization: T.P., D.O., C.R.K., F.Z., and I.A.W.; Investigation: T.P., D.O., Y.F.-G. G.G.-P., Z. H., and K.L.W.; Resources: G.G.-P., W.V., D.E., E.L., and C.R.K.; Formal analysis T.P., D.O., Y.F.-G., F.Z., and I.A.W.; Writing-original draft preparation: T.P., D.O., and I.A.W.; Writing-reviewing and editing: all authors.

\section{Competing interests}

The authors T.P., D.O., Y.F.G., G.G.-P., Z.H., E.L., C.R.K., F.Z., and I.A.W. declare that they have no competing interests. K.L.W., W.V., and P.K. are employees of and own equity in Atreca, Inc.

\section{Additional information}

Supplementary information The online version contains supplementary material available at https://doi.org/10.1038/s41467-021-21221-4.

Correspondence and requests for materials should be addressed to I.A.W.

Peer review information Nature Communications thanks the anonymous reviewer(s) for their contribution to the peer review of this work. Peer reviewer reports are available.

Reprints and permission information is available at http://www.nature.com/reprints

Publisher's note Springer Nature remains neutral with regard to jurisdictional claims in published maps and institutional affiliations.

Open Access This article is licensed under a Creative Commons Attribution 4.0 International License, which permits use, sharing, adaptation, distribution and reproduction in any medium or format, as long as you give appropriate credit to the original author(s) and the source, provide a link to the Creative Commons license, and indicate if changes were made. The images or other third party material in this article are included in the article's Creative Commons license, unless indicated otherwise in a credit line to the material. If material is not included in the article's Creative Commons license and your intended use is not permitted by statutory regulation or exceeds the permitted use, you will need to obtain permission directly from the copyright holder. To view a copy of this license, visit http://creativecommons.org/ licenses/by/4.0/

(C) The Author(s) 2021 